\title{
Compact hyperbolic Coxeter $n$-polytopes with $n+3$ facets
}

\author{
Pavel Tumarkin* \\ Independent University of Moscow \\ B. Vlassievskii 11, 119002 Moscow, Russia \\ pasha@mccme.ru
}

Submitted: Apr 23, 2007; Accepted: Sep 30, 2007; Published: Oct 5, 2007

Mathematics Subject Classifications: 51M20, 51F15, 20F55

\begin{abstract}
We use methods of combinatorics of polytopes together with geometrical and computational ones to obtain the complete list of compact hyperbolic Coxeter $n$ polytopes with $n+3$ facets, $4 \leq n \leq 7$. Combined with results of Esselmann this gives the classification of all compact hyperbolic Coxeter $n$-polytopes with $n+3$ facets, $n \geq 4$. Polytopes in dimensions 2 and 3 were classified by Poincaré and Andreev.
\end{abstract}

\section{Introduction}

A polytope in the hyperbolic space $\mathbb{H}^{n}$ is called a Coxeter polytope if its dihedral angles are all integer submultiples of $\pi$. Any Coxeter polytope $P$ is a fundamental domain of the discrete group generated by reflections in the facets of $P$.

There is no complete classification of compact hyperbolic Coxeter polytopes. Vinberg [V1] proved there are no such polytopes in $\mathbb{H}^{n}, n \geq 30$. Examples are known only for $n \leq 8$ (see [B1], [B2]).

In dimensions 2 and 3 compact Coxeter polytopes were completely classified by Poincaré $[\mathrm{P}]$ and Andreev $[\mathrm{A}]$. Compact polytopes of the simplest combinatorial type, the simplices, were classified by Lannér [L]. Kaplinskaja [K] (see also [V2]) listed simplicial prisms, Esselmann [E2] classified the remaining compact $n$-polytopes with $n+2$ facets.

In the paper $[\operatorname{ImH}] \mathrm{Im}$ Hof classified polytopes that can be described by Napier cycles. These polytopes have at most $n+3$ facets. Concerning polytopes with $n+3$ facets, Esselmann proved the following theorem ([E1, Th. 5.1]):

*Partially supported by grants MK-6290.2006.1, NSh-5666.2006.1, INTAS grant YSF-06-100000145916, and RFBR grant 07-01-00390-a 
Let $P$ be a compact hyperbolic Coxeter n-polytope bounded by $n+3$ facets. Then $n \leq 8$; if $n=8$, then $P$ is the polytope found by Bugaenko in [B2]. This polytope has the following Coxeter diagram:

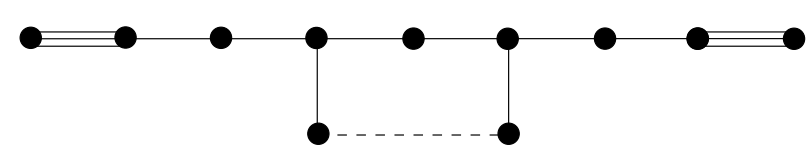

In this paper, we expand the technique derived by Esselmann in [E1] and [E2] to complete the classification of compact hyperbolic Coxeter $n$-polytopes with $n+3$ facets. The aim is to prove the following theorem:

Main Theorem. Tables 4.8-4.11 contain all Coxeter diagrams of compact hyperbolic Coxeter n-polytopes with $n+3$ facets for $n \geq 4$.

The paper is organized as follows. In Section 2 we recall basic definitions and list some well-known properties of hyperbolic Coxeter polytopes. We also emphasize the connection between combinatorics (Gale diagram) and metric properties (Coxeter diagram) of hyperbolic Coxeter polytope. In Section 3 we recall some technical tools from [V1] and [E1] concerning Coxeter diagrams and Gale diagrams, and introduce notation suitable for investigating of large number of diagrams. Section 4 is devoted to the proof of the main theorem. The most part of the proof is computational: we restrict the number of Coxeter diagrams in consideration, and use a computer check after that. The bulk is to find an upper bound for the number of diagrams, and then to reduce the number to make the computation short enough.

This paper is a completely rewritten part of my Ph.D. thesis (2004) with several errors corrected. I am grateful to my advisor Prof. E. B. Vinberg for his help. I am also grateful to Prof. R. Kellerhals who brought the papers of F. Esselmann and L. Schlettwein to my attention, and to the referee for useful suggestions.

\section{Hyperbolic Coxeter polytopes and Gale diagrams}

In this section we list essential facts concerning hyperbolic Coxeter polytopes, Gale diagrams of simple polytopes, and Coxeter diagrams we use in this paper. Proofs, details and definitions in general case may be found in [G] and [V2]. In the last part of this section we present the main tools used for the proof of the main theorem.

We write $n$-polytope instead of " $n$-dimensional polytope" for short. By facet we mean a face of codimension one.

\subsection{Gale diagrams}

An $n$-polytope is called simple if any its $k$-face belongs to exactly $n-k$ facets. Proposition 2.2 implies that any compact hyperbolic Coxeter polytope is simple. From now on we consider simple polytopes only. 
Every combinatorial type of simple $n$-polytope with $d$ facets can be represented by its Gale diagram $G$. This consists of $d$ points $a_{1}, \ldots, a_{d}$ on the $(d-n-2)$-dimensional unit sphere in $\mathbb{R}^{d-n-1}$ centered at the origin.

The combinatorial type of a simple convex polytope can be read off from the Gale diagram in the following way. Each point $a_{i}$ corresponds to the facet $f_{i}$ of $P$. For any subset $J$ of the set of facets of $P$ the intersection of facets $\left\{f_{j} \mid j \in J\right\}$ is a face of $P$ if and only if the origin is contained in the interior of $\operatorname{conv}\left\{a_{j} \mid j \notin J\right\}$.

The points $a_{1}, \ldots, a_{d} \in \mathbb{S}^{d-n-2}$ compose a Gale diagram of some $n$-dimensional polytope $P$ with $d$ facets if and only if every open half-space $H^{+}$in $\mathbb{R}^{d-n-1}$ bounded by a hyperplane $H$ through the origin contains at least two of the points $a_{1}, \ldots, a_{d}$.

We should notice that the definition of Gale diagram introduced above is "dual" to the standard one (see, for example, $[G]$ ): usually Gale diagram is defined in terms of vertices of polytope instead of facets. Notice also that the definition above concerns simple polytopes only, and it takes simplices out of consideration: usually one means the origin of $\mathbb{R}^{1}$ with multiplicity $n+1$ by the Gale diagram of an $n$-simplex, however we exclude the origin since we consider simple polytopes only, and the origin is not contained in $G$ for any simple polytope except simplex.

We say that two Gale diagrams $G$ and $G^{\prime}$ are isomorphic if the corresponding polytopes are combinatorially equivalent.

If $d=n+3$ then the Gale diagram of $P$ is two-dimensional, i.e. nodes $a_{i}$ of the diagram lie on the unit circle.

A standard Gale diagram of simple $n$-polytope with $n+3$ facets consists of vertices $v_{1}, \ldots, v_{k}$ of regular $k$-gon ( $k$ is odd) in $\mathbb{R}^{2}$ centered at the origin which are labeled according to the following rules:

1) Each label is a positive integer, the sum of labels equals $n+3$.

2) The vertices that lie in any open half-space bounded by a line through the origin have labels whose sum is at least two.

Each point $v_{i}$ with label $\mu_{i}$ corresponds to $\mu_{i}$ facets $f_{i, 1}, \ldots, f_{i, \mu_{i}}$ of $P$. For any subset $J$ of the set of facets of $P$ the intersection of facets $\left\{f_{j, \gamma} \mid(j, \gamma) \in J\right\}$ is a face of $P$ if and only if the origin is contained in the interior of $\operatorname{conv}\left\{v_{j} \mid(j, \gamma) \notin J\right\}$.

It is easy to check (see, for example, [G, Sec. 6.3]) that any two-dimensional Gale diagram is isomorphic to some standard diagram. Two simple $n$-polytopes with $n+3$ facets are combinatorially equivalent if and only if their standard Gale diagrams are congruent.

\subsection{Coxeter diagrams}

Any Coxeter polytope $P$ can be represented by its Coxeter diagram.

An abstract Coxeter diagram is a one-dimensional simplicial complex with weighted edges, where weights are either of the type $\cos \frac{\pi}{m}$ for some integer $m \geq 3$ or positive real numbers no less than one. We can suppress the weights but indicate the same information by labeling the edges of a Coxeter diagram in the following way: 
- if the weight equals $\cos \frac{\pi}{m}$ then the nodes are joined by either an $(m-2)$-fold edge or a simple edge labeled by $m$;

- if the weight equals one then the nodes are joined by a bold edge;

- if the weight is greater than one then the nodes are joined by a dotted edge labeled by its weight.

A subdiagram of Coxeter diagram is a subcomplex with the same as in $\Sigma$. The order $|\Sigma|$ is the number of vertices of the diagram $\Sigma$.

If $\Sigma_{1}$ and $\Sigma_{2}$ are subdiagrams of a Coxeter diagram $\Sigma$, we denote by $\left\langle\Sigma_{1}, \Sigma_{2}\right\rangle$ a subdiagram of $\Sigma$ spanned by all nodes of $\Sigma_{1}$ and $\Sigma_{2}$. We say that a node of $\Sigma$ attaches to a subdiagram $\Sigma_{1} \subset \Sigma$ if it is joined with some nodes of $\Sigma_{1}$ by edges of any type.

Let $\Sigma$ be a diagram with $d$ nodes $u_{1}, \ldots, u_{d}$. Define a symmetric $d \times d$ matrix $\operatorname{Gr}(\Sigma)$ in the following way: $g_{i i}=1$; if two nodes $u_{i}$ and $u_{j}$ are adjacent then $g_{i j}$ equals negative weight of the edge $u_{i} u_{j}$; if two nodes $u_{i}$ and $u_{j}$ are not adjacent then $g_{i j}$ equals zero.

By signature and determinant of diagram $\Sigma$ we mean the signature and the determinant of the matrix $\operatorname{Gr}(\Sigma)$.

An abstract Coxeter diagram $\Sigma$ is called elliptic if the matrix $\operatorname{Gr}(\Sigma)$ is positive definite. A Coxeter diagram $\Sigma$ is called parabolic if the matrix $\operatorname{Gr}(\Sigma)$ is degenerate, and any subdiagram of $\Sigma$ is elliptic. Connected elliptic and parabolic diagrams were classified by Coxeter $[\mathrm{C}]$. We represent the list in Table 2.1 .

A Coxeter diagram $\Sigma$ is called a Lannér diagram if any subdiagram of $\Sigma$ is elliptic, and the diagram $\Sigma$ is neither elliptic nor parabolic. Lannér diagrams were classified by Lannér $[\mathrm{L}]$. We represent the list in Table 2.2. A diagram $\Sigma$ is superhyperbolic if its negative inertia index is greater than 1 .

By a simple (resp., multiple) edge of Coxeter diagram we mean an $(m-2)$-fold edge where $m$ is equal to (resp., greater than) 3. The number $m-2$ is called the multiplicity of a multiple edge. Edges of multiplicity greater than 3 we call multi-multiple edges. If an edge $u_{i} u_{j}$ has multiplicity $m-2$ (i.e. the corresponding facets form an angle $\frac{\pi}{m}$ ), we write $\left[u_{i}, u_{j}\right]=m$.

A Coxeter diagram $\Sigma(P)$ of Coxeter polytope $P$ is a Coxeter diagram whose matrix $\operatorname{Gr}(\Sigma)$ coincides with Gram matrix of outer unit normals to the facets of $P$ (referring to the standard model of hyperbolic $n$-space in $\mathbb{R}^{n, 1}$ ). In other words, nodes of Coxeter diagram correspond to facets of $P$. Two nodes are joined by either an $(m-2)$-fold edge or an $m$-labeled edge if the corresponding dihedral angle equals $\frac{\pi}{m}$. If the corresponding facets are parallel the nodes are joined by a bold edge, and if they diverge then the nodes are joined by a dotted edge (which may be labeled by hyperbolic cosine of distance between the hyperplanes containing these facets).

If $\Sigma(P)$ is the Coxeter diagram of $P$ then nodes of $\Sigma(P)$ are in one-to-one correspondence with elements of the set $I=\{1, \ldots, d\}$. For any subset $J \subset I$ denote by $\Sigma(P)_{J}$ the subdiagram of $\Sigma(P)$ that consists of nodes corresponding to elements of $J$. 
Table 2.1: Connected elliptic and parabolic Coxeter diagrams are listed in left and right columns respectively.

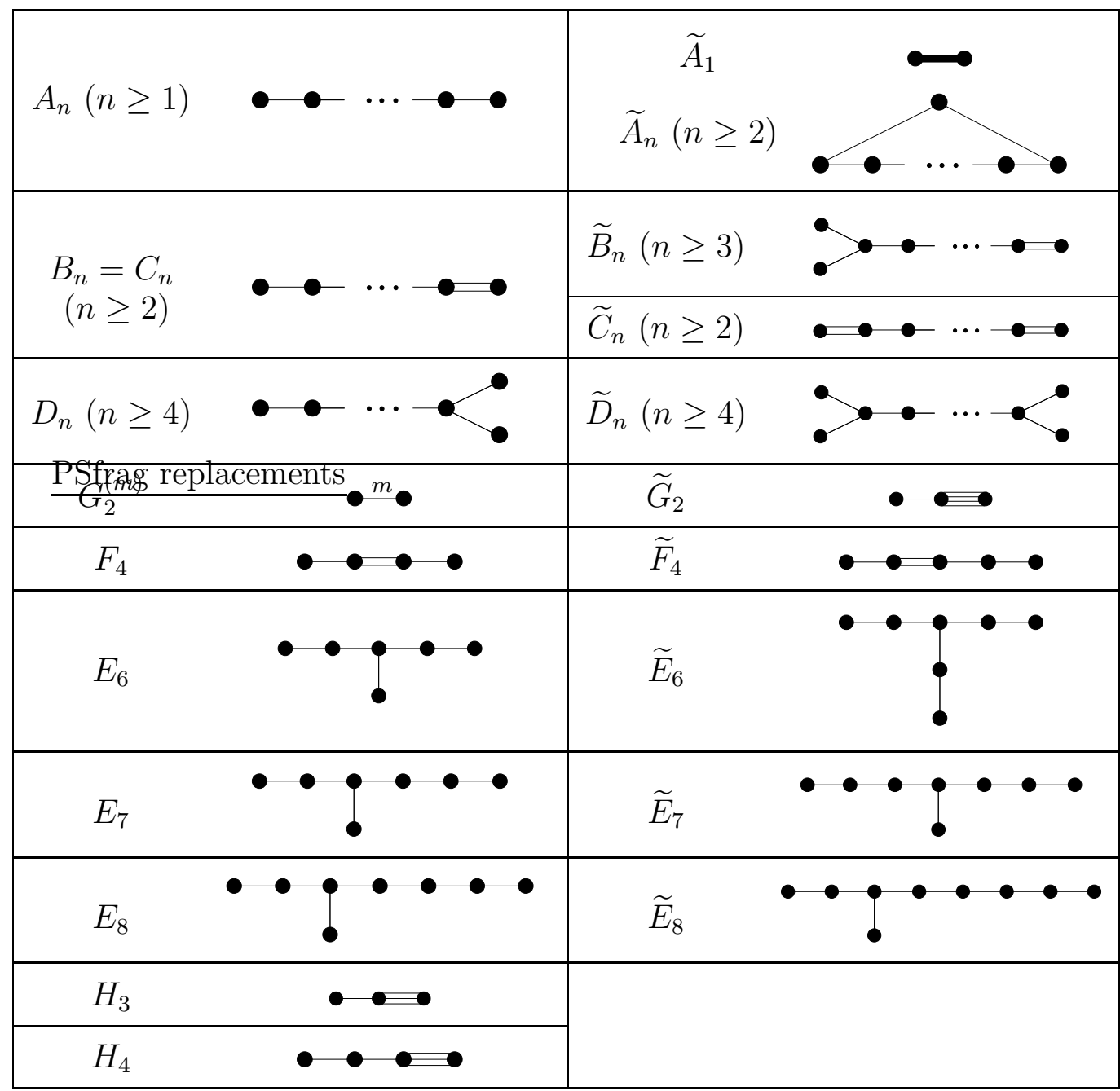

\subsection{Hyperbolic Coxeter polytopes}

In this section by polytope we mean a (probably non-compact) intersection of closed half-spaces.

Proposition 2.1 ([V2], Th. 2.1). Let $\mathrm{Gr}=\left(g_{i j}\right)$ be indecomposable symmetric matrix of signature $(n, 1)$, where $g_{i i}=1$ and $g_{i j} \leq 0$ if $i \neq j$. Then there exists a unique (up to isometry of $\left.\mathbb{H}^{n}\right)$ convex polytope $P \subset \mathbb{H}^{n}$ whose Gram matrix coincides with $\mathrm{Gr}$.

Let $\mathrm{Gr}$ be the Gram matrix of the polytope $P$, and let $J \subset I$ be a subset of the set of facets of $P$. Denote by $\operatorname{Gr}_{J}$ the Gram matrix of vectors $\left\{e_{i} \mid i \in J\right\}$, where $e_{i}$ is outward unit normal to the facet $f_{i}$ of $P$ (i.e. $\operatorname{Gr}_{J}=\operatorname{Gr}\left(\Sigma(P)_{J}\right)$ ). Denote by $|J|$ the number of elements of $J$. 
Table 2.2: Lannér diagrams.

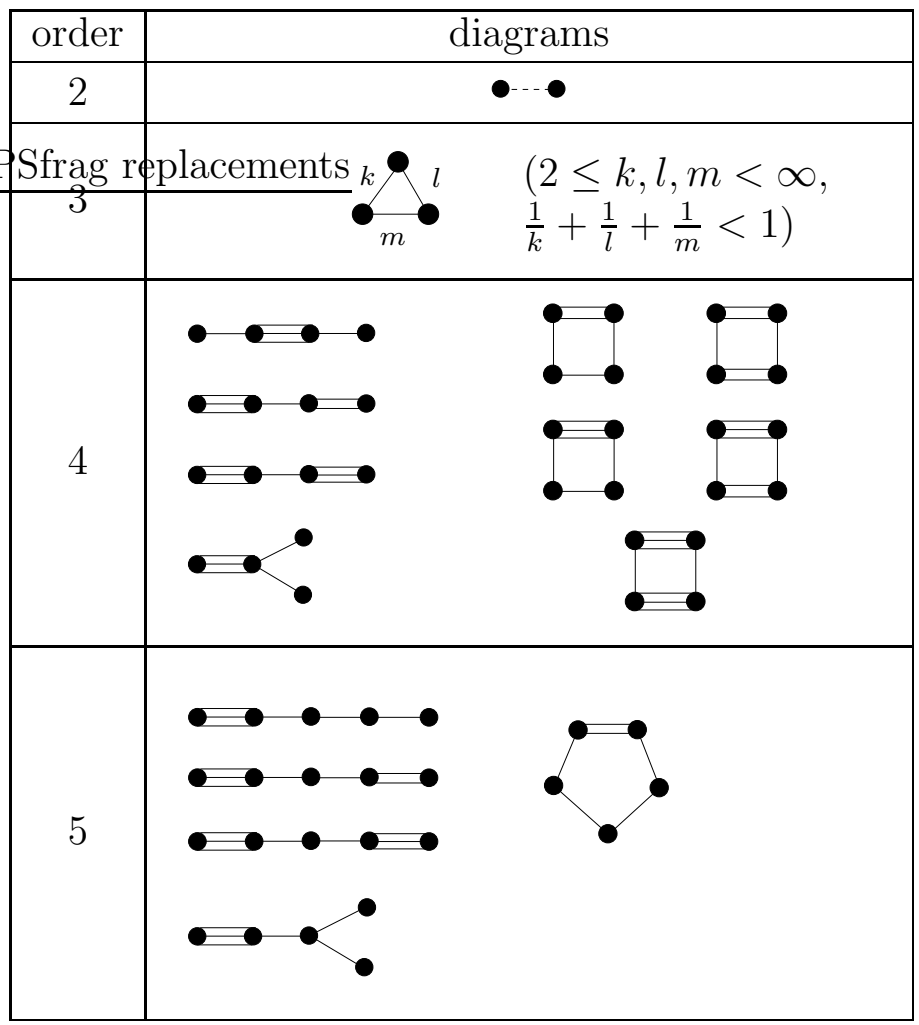

Proposition 2.2 ([V2], Th. 3.1). Let $P \subset \mathbb{H}^{n}$ be an acute-angled polytope with Gram matrix Gr, and let $J$ be a subset of the set of facets of $P$. The set

$$
q=P \cap \bigcap_{i \in J} f_{i}
$$

is a face of $P$ if and only if the matrix $\mathrm{Gr}_{J}$ is positive definite. Dimension of $q$ is equal to $n-|J|$.

Notice that Prop. 2.2 implies that the combinatorics of $P$ is completely determined by the Coxeter diagram $\Sigma(P)$.

Let $A$ be a symmetric matrix whose non-diagonal elements are non-positive. $A$ is called indecomposable if it cannot be transformed to a block-diagonal matrix via simultaneous permutations of columns and rows. We say $A$ to be parabolic if any indecomposable component of $A$ is positive semidefinite and degenerate. For example, a matrix $\operatorname{Gr}(\Sigma)$ for any parabolic diagram $\Sigma$ is parabolic.

Proposition 2.3 ([V2], cor. of Th. 4.1, Prop. 3.2 and Th. 3.2). Let $P \subset \mathbb{H}^{n}$ be a compact Coxeter polytope, and let Gr be its Gram matrix. Then for any $J \subset I$ the matrix $\mathrm{Gr}_{J}$ is not parabolic. 
Corollary 2.1 reformulates Prop. 2.3 in terms of Coxeter diagrams.

Corollary 2.1. Let $P \subset \mathbb{H}^{n}$ be a compact Coxeter polytope, and let $\Sigma$ be its Coxeter matrix. Then any non-elliptic subdiagram of $\Sigma$ contains a Lannér subdiagram.

Proposition 2.4 ([V2], Prop. 4.2). A polytope $P$ in $\mathbb{H}^{n}$ is compact if and only if it is combinatorially equivalent to some compact convex n-polytope.

The main result of paper [FT] claims that if $P$ is a compact hyperbolic Coxeter $n$ polytope having no pair of disjoint facets, then $P$ is either a simplex or one of the seven polytopes with $n+2$ facets described in [E1]. As a corollary, we obtain the following proposition.

Proposition 2.5. Let $P \subset \mathbb{H}^{n}$ be a compact Coxeter polytope with at least $n+3$ facets. Then $P$ has a pair of disjoint facets.

\subsection{Coxeter diagrams, Gale diagrams, and missing faces}

Now, for any compact hyperbolic Coxeter polytope we have two diagrams which carry the complete information about its combinatorics, namely Gale diagram and Coxeter diagram. The interplay between them is described by the following lemma, which is a reformulation of results listed in Section 2.3 in terms of Coxeter diagrams and Gale diagrams.

Lemma 2.1. A Coxeter diagram $\Sigma$ with nodes $\left\{u_{i} \mid i=1, \ldots, d\right\}$ is a Coxeter diagram of some compact hyperbolic Coxeter n-polytope with $d$ facets if and only if the following two conditions hold:

1) $\Sigma$ is of signature $(n, 1, d-n-1)$;

2) there exists a $(d-n-1)$-dimensional Gale diagram with nodes $\left\{v_{i} \mid i=1, \ldots, d\right\}$ and one-to-one map $\psi:\left\{u_{i} \mid i=1, \ldots, d\right\} \rightarrow\left\{v_{i} \mid i=1, \ldots, d\right\}$ such that for any $J \subset$ $\{1, \ldots, d\}$ the subdiagram $\Sigma_{J}$ of $\Sigma$ is elliptic if and only if the origin is contained in the interior of $\operatorname{conv}\left\{\psi\left(v_{i}\right) \mid i \notin J\right\}$.

Let $P$ be a simple polytope. The facets $f_{1}, \ldots, f_{m}$ of $P$ compose a missing face of $P$ if $\bigcap_{i=1}^{m} f_{i}=\emptyset$ but any proper subset of $\left\{f_{1}, \ldots, f_{m}\right\}$ has a non-empty intersection.

Proposition 2.6 ([FT], Lemma 2). Let $P$ be a simple $d$-polytope with $d+k$ facets $\left\{f_{i}\right\}$, let $G=\left\{a_{i}\right\} \subset \mathbb{S}^{k-2}$ be a Gale diagram of $P$, and let $I \subset\{1, \ldots, d+k\}$. Then the set $M_{I}=\left\{f_{i} \mid i \in I\right\}$ is a missing face of $P$ if and only if the following two conditions hold:

(1) there exists a hyperplane $H$ through the origin separating the set $\widehat{M}_{I}=\left\{a_{i} \mid i \in I\right\}$ from the remaining points of $G$;

(2) for any proper subset $J \subset I$ no hyperplane through the origin separates the set $\widehat{M}_{J}=\left\{a_{i} \mid i \in J\right\}$ from the remaining points of $G$. 
Remark. Suppose that $P$ is a compact hyperbolic Coxeter polytope. The definition of missing face (together with Cor. 2.1) implies that for any Lannér subdiagram $L \subset \Sigma(P)$ the facets corresponding to $L$ compose a missing face of $P$, and any missing face of $P$ corresponds to some Lannér diagram in $\Sigma(P)$.

Now consider a compact hyperbolic Coxeter $n$-polytope $P$ with $n+3$ facets with standard Gale diagram $G$ (which is a $k$-gon, $k$ is odd) and Coxeter diagram $\Sigma$. Denote by $\Sigma_{i, j}$ a subdiagram of $\Sigma$ corresponding to $j-i+1(\bmod k)$ consecutive nodes $a_{i}, \ldots, a_{j}$ of $G$ (in the sense of Lemma 2.1). If $i=j$, denote $\Sigma_{i, i}$ by $\Sigma_{i}$.

The following lemma is an immediate corollary of Prop. 2.6.

Lemma 2.2. For any $i \in\{0, \ldots, k-1\}$ a diagram $\Sigma_{i+1, i+\frac{k-1}{2}}$ is a Lannér diagram. All Lannér diagrams contained in $\Sigma$ are of this type.

It is easy to see that the collection of missing faces completely determines the combinatorics of $P$. In view of Lemma 2.2 and the remark above, this means that in Lemma 2.1 for given Coxeter diagram we need to check the signature and correspondence of Lannér diagrams to missing faces of some Gale diagram.

Example. Suppose that there exists a compact hyperbolic Coxeter polytope $P$ with standard Gale diagram $G$ shown in Fig. 2.1(a). What can we say about Coxeter diagram $\Sigma=\Sigma(P)$ ?

(a)

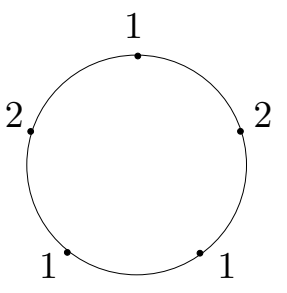

(b)

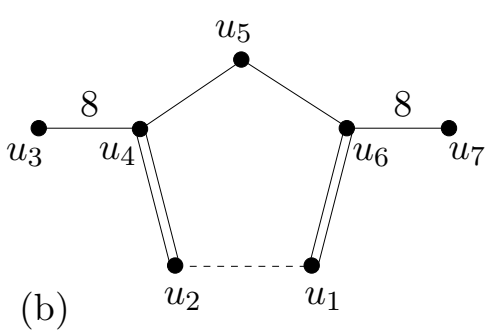

$u_{2} \quad u_{1}$

Figure 2.1: (a) A standard Gale diagram $G$ and (b) a Coxeter diagram of one of polytopes with Gale diagram $G$

The sum of labels of nodes of Gale diagram $G$ is equal to 7 , so $P$ is a 4-polytope with 7 facets. Thus, $\Sigma$ is spanned by nodes $u_{1}, \ldots, u_{7}$, and its signature equals $(4,1,2)$. Further, $G$ is a pentagon. By Lemma $2.2, \Sigma$ contains exactly 5 Lannér diagrams, namely $\left\langle u_{1}, u_{2}\right\rangle$, $\left\langle u_{2}, u_{3}, u_{4}\right\rangle,\left\langle u_{3}, u_{4}, u_{5}\right\rangle,\left\langle u_{5}, u_{6}, u_{7}\right\rangle$, and $\left\langle u_{6}, u_{7}, u_{1}\right\rangle$.

Now consider the Coxeter diagram $\Sigma$ shown in Fig. 2.1(b). Assigning label $1+\sqrt{2}$ to the dotted edge of $\Sigma$, we obtain a diagram of signature $(4,1,2)$ (this may be shown by direct calculation). Therefore, there exist 7 vectors in $\mathbb{H}^{4}$ with Gram matrix $\operatorname{Gr}(\Sigma)$. It is easy to see that $\Sigma$ contains exactly 5 Lannér diagrams described above. Thus, $\Sigma$ is a Coxeter diagram of some compact 4-polytope with Gale diagram $G$.

Of course, $\Sigma$ is just an example of a Coxeter diagram satisfying both conditions of Lemma 2.1 with respect to given Gale diagram $G$. In the next two sections we will show how to list all compact hyperbolic Coxeter polytopes of given combinatorial type. 


\section{Technical tools}

From now on by polytope we mean a compact hyperbolic Coxeter $n$-polytope with $n+3$ facets, and we deal with standard Gale diagrams only.

\subsection{Admissible Gale diagrams}

Suppose that there exists a compact hyperbolic Coxeter polytope $P$ with $k$-angled Gale diagram $G$. Since the maximal order of Lannér diagram equals five, Lemma 2.2 implies that the sum of labels of $\frac{k-1}{2}$ consecutive nodes of Gale diagram does not exceed five. On the other hand, by Lemma $2.5, P$ has a missing face of order two. This is possible in two cases only: either $G$ is a pentagon with two neighboring vertices labeled by 1 , or $G$ is a triangle one of whose vertices is labeled by 2 (see Prop. 2.6). Table 3.1 contains all Gale diagrams satisfying one of two conditions above with at least 7 and at most 10 vertices, i.e. Gale diagrams that may correspond to compact hyperbolic Coxeter $n$-polytopes with $n+3$ facets for $4 \leq n \leq 7$.

\subsection{Admissible arcs}

Let $P$ be an $n$-polytope with $n+3$ facets and let $G$ be its $k$-angled Gale diagram. By Lemma 2.2 , for any $i \in\{0, \ldots, k-1\}$ the diagram $\Sigma_{i+1, i+\frac{k-1}{2}}$ is a Lannér diagram. Denote by

$$
\left\lfloor x_{1}, \ldots, x_{l}\right\rfloor_{\frac{k-1}{2}}, \quad l \leq k
$$

an arc of length $l$ of $G$ that consists of $l$ consecutive nodes with labels $x_{1}, \ldots, x_{l}$. By writing $J=\left\lfloor x_{1}, \ldots, x_{l}\right\rfloor_{\frac{k-1}{2}}$ we mean that $J$ is the set of facets of $P$ corresponding to these nodes of $G$. The index $\frac{k-1}{2}$ means that for any $\frac{k-1}{2}$ consecutive nodes of the arc (i.e. for any arc $\left.I=\left\lfloor x_{i+1}, \ldots, x_{i+\frac{k-1}{2}}\right\rfloor_{\frac{k-1}{2}}\right)$ the subdiagram $\Sigma_{I}$ of $\Sigma(P)$ corresponding to these nodes is a Lannér diagram (i.e. $I$ is a missing face of $P$ ).

By Cor. 2.1, any diagram $\Sigma_{J} \subset \Sigma(P)$ corresponding to an arc $J=\left\lfloor x_{1}, \ldots, x_{l}\right\rfloor_{\frac{k-1}{2}}$ satisfies the following property: any subdiagram of $\Sigma_{J}$ containing no Lannér diagram is elliptic. Clearly, any subdiagram of $\Sigma(P)$ containing at least one Lannér diagram is of signature $(k, 1)$ for some $k \leq n$. As it is shown in [E1], for some arcs $J$ there exist a few corresponding diagrams $\Sigma_{J}$ only. In the following lemma, we recall some results of Esselmann [E1] and prove similar facts concerning some arcs of Gale diagrams listed in Table 3.1. This will help us to restrict the number of Coxeter diagrams that may correspond to some of Gale diagrams listed in Table 3.1.

Lemma 3.1. The diagrams presented in the middle column of Table 3.2 are the only diagrams that may correspond to arcs listed in the left column.

Proof. At first, notice that for any $J$ as above (i.e. $J$ consists of several consecutive nodes of Gale diagram) the diagram $\Sigma_{J}$ must be connected. This follows from the fact that any Lannér diagram is connected, and that $\Sigma_{J}$ is not superhyperbolic. 
Table 3.1: Gale diagrams that may correspond to compact Coxeter polytopes (see Section 3.1)

$\underline{n=4}$
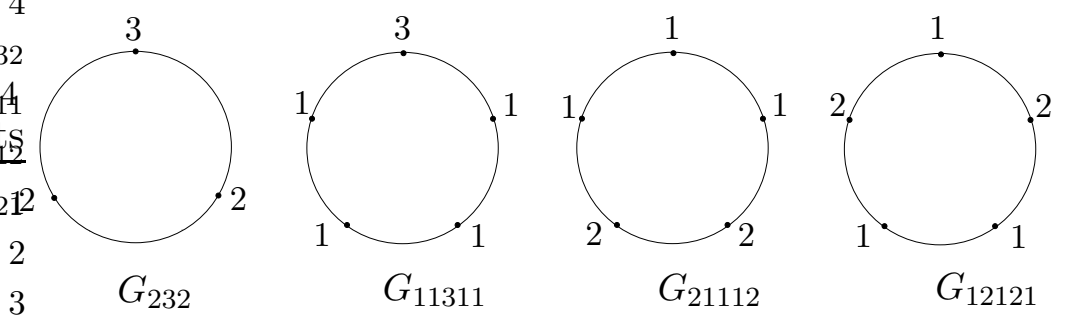

$\underline{n=5}$
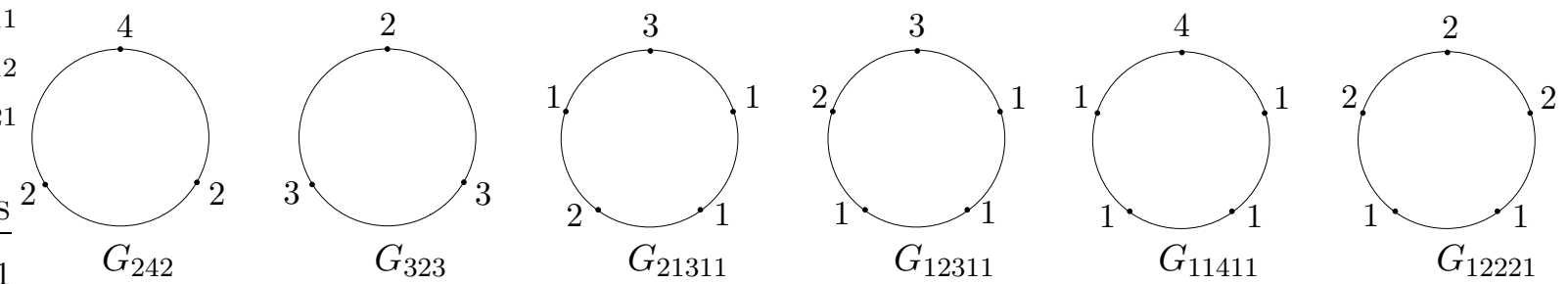

$\underline{n=6}$
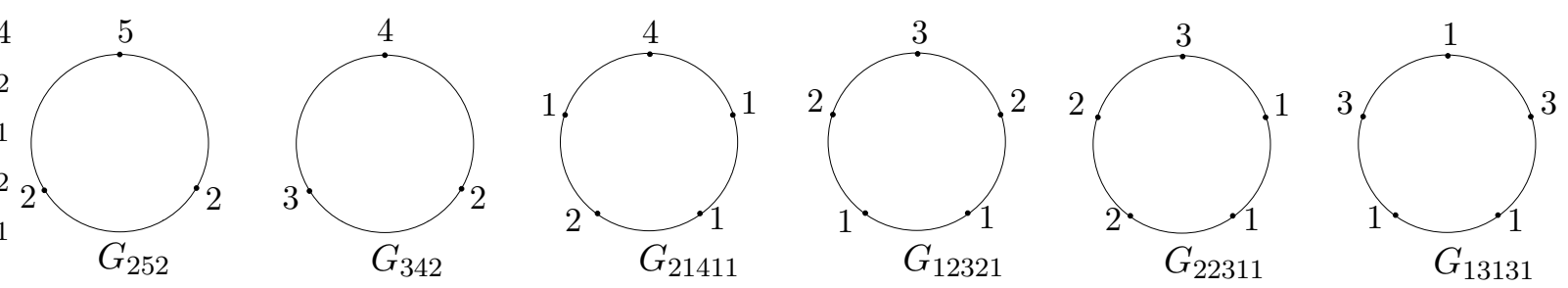

$\underline{n=7}$
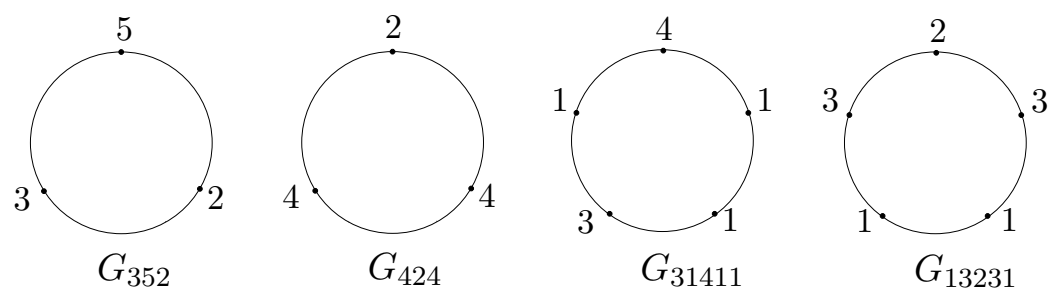

Now we restrict our considerations to items 8-11 only. For none of these $J$ the diagram $\Sigma_{J}$ contains a Lannér diagram of order 2 or 3 . Since $\Sigma_{J}$ is connected and does not contain parabolic subdiagrams, this implies that $\Sigma_{J}$ does not contain neither dotted nor multimultiple edges. Thus, we are left with finitely many possibilities only, that allows us to use a computer check: there are several (from 5 to 7 ) nodes, some of them joined by edges of multiplicity at most 3 . We only need to check all possible diagrams for the number of 
Table 3.2: Possible diagrams $\Sigma_{J}$ for some $\operatorname{arcs} J$. White nodes correspond to endpoints of arcs having multiplicity one

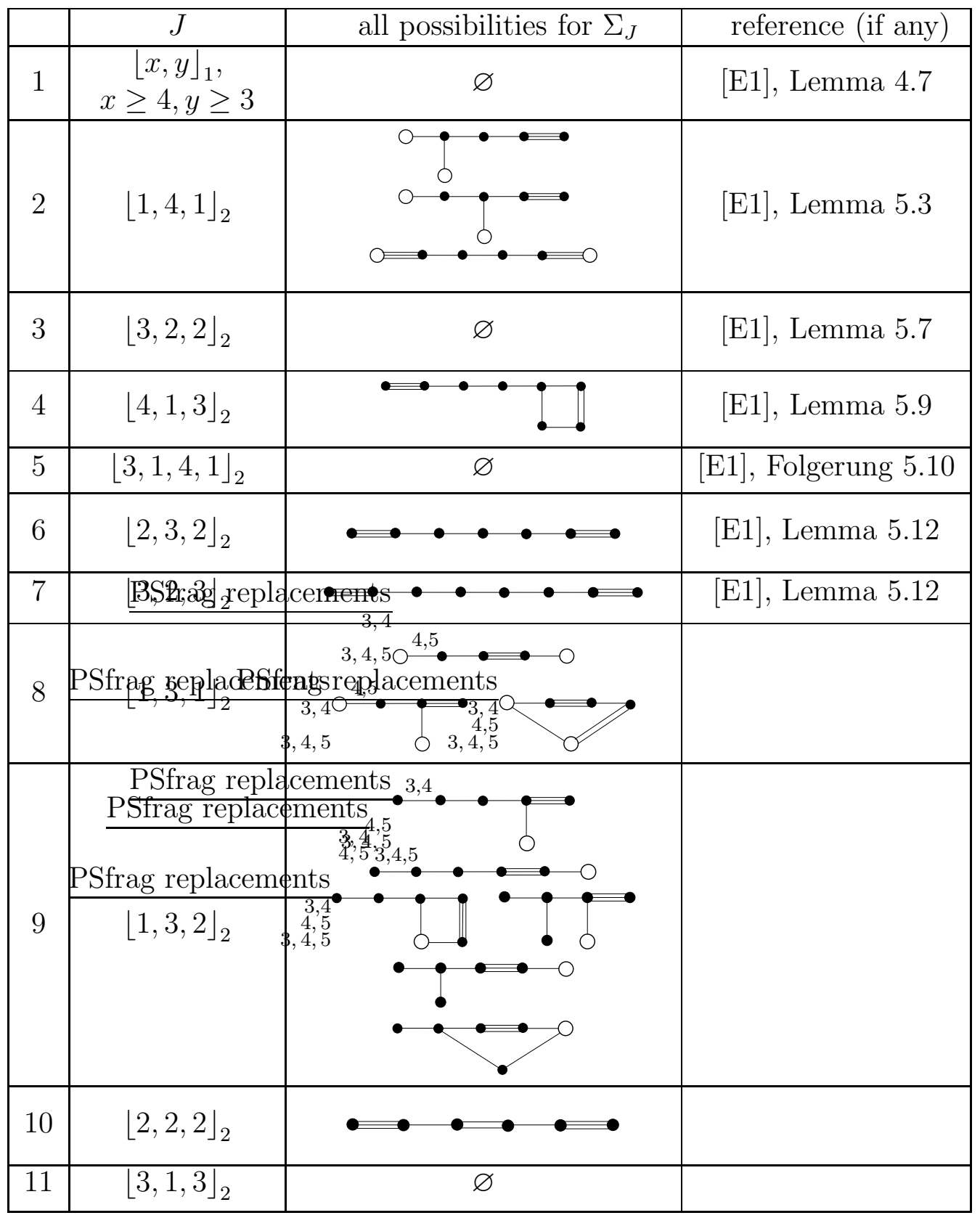

Lannér diagrams of all orders and for parabolic subdiagrams. Namely, in items 8, 10 and 11 we look for diagrams of order 5, 6 and 7 containing exactly 2 Lannér subdiagrams of order 4 (and containing neither other Lannér diagrams nor parabolic subdiagrams), and in 
item 9 we look for diagrams of order 6 containing exactly one Lannér subdiagram of order 4 and exactly one Lannér diagram of order 5 . Notice also that we do not need to check the signature of obtained diagrams: all them are certainly non-elliptic, and since any of them contains exactly two Lannér diagrams which have at least one node in common, by excluding this node we obtain an elliptic diagram.

However, the computation described above is really huge. In what follows we describe case-by-case how to reduce these computations to a few minutes of hand-calculations.

- Item $8\left(J=\lfloor 1,3,1\rfloor_{2}\right)$. We may consider $\Sigma_{J}$ as a Lannér diagram $L$ of order 4 together with one vertex attached to $L$ to compose a unique additional Lannér diagram which should be of order 4 , too. There are 9 possibilities for $L$ only (Table 2.2).

- Item $9\left(J=\lfloor 1,3,2\rfloor_{2}\right)$. The considerations follow the preceding ones, but we take as $L$ a Lannér diagram of order 5 . Again, there are few possibilities for $L$ only (namely five: see Table 2.2).

- Item $10\left(J=\lfloor 2,2,2\rfloor_{2}\right)$. Again, $\Sigma_{J}$ contains a Lannér diagram $L$ of order 4 . One of the two remaining nodes of $\Sigma_{J}$ must be attached to $L$. Denote this node by $v$. The diagram $\langle L, v\rangle \subset \Sigma_{J}$ consists of five nodes and contains a unique Lannér diagram which is of order 4. All such diagrams are listed in [E1, Lemma 3.8] (see the first two rows of Tabelle 3, the case $\left.\left|\mathcal{N}_{F}\right|=1,\left|\mathcal{L}_{F}\right|=4\right)$. We reproduce this list in Table 3.3.

Table 3.3: One of these diagrams should be contained in $\Sigma_{J}$ for $J=\lfloor 2,2,2\rfloor_{2}$
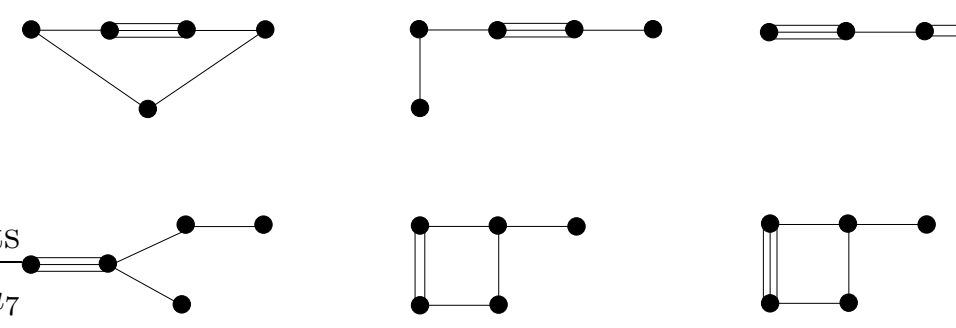

One can see that there are six possibilities only. Now to each of them we attach the remaining node to compose a unique new Lannér diagram which should be of order 4 .

- Item $11\left(J=\lfloor 3,1,3\rfloor_{2}\right)$. The considerations are very similar to the preceding case. $\Sigma_{J}$ contains a Lannér diagram $L$ of order 4 . One of the three remaining nodes of $\Sigma_{J}$ must be attached to $L$. Denote this node by $v$. Now, one of the two remaining nodes attaches to $\langle L, v\rangle \subset \Sigma_{J}$. Denote it by $u$. The diagram $\langle L, v, u\rangle \subset \Sigma_{J}$ consists of six nodes and contains a unique Lannér diagram which is of order 4. All such diagrams are listed in [E1, Lemma 3.8] (see Tabelle 3, the first two rows of page 27, the case $\left|\mathcal{N}_{F}\right|=2,\left|\mathcal{L}_{F}\right|=4$ ). We reproduce this list in Table 3.4.

There are five possibilities only. As above, we attach to each of them the remaining node to compose a unique new Lannér diagram which should be of order 4 . 
Table 3.4: One of these diagrams should be contained in $\Sigma_{J}$ for $J=\lfloor 3,1,3\rfloor_{2}$
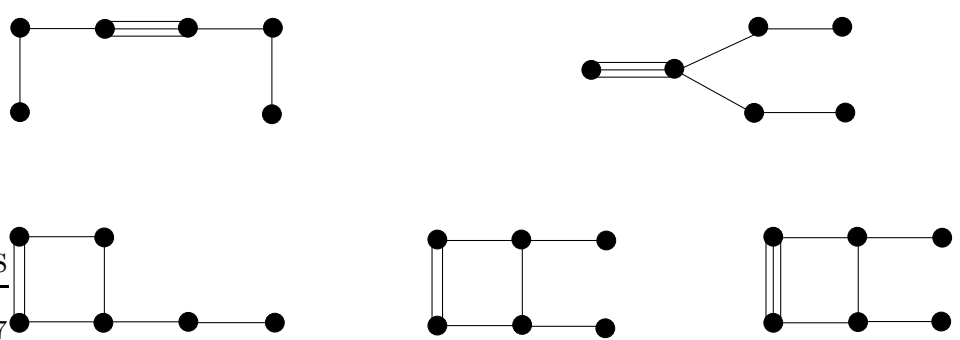

\subsection{Local determinants}

In this section we list some tools derived in [V1] to compute determinants of Coxeter diagrams. We will use them to show that some (infinite) series of Coxeter diagrams are superhyperbolic.

Let $\Sigma$ be a Coxeter diagram, and let $T$ be a subdiagram of $\Sigma$ such that $\operatorname{det}(\Sigma \backslash T) \neq 0$. A local determinant of $\Sigma$ on a subdiagram $T$ is

$$
\operatorname{det}(\Sigma, T)=\frac{\operatorname{det} \Sigma}{\operatorname{det}(\Sigma \backslash T)}
$$

Proposition 3.1 ([V1], Prop. 12). If a Coxeter diagram $\Sigma$ consists of two subdiagrams $\Sigma_{1}$ and $\Sigma_{2}$ having a unique vertex $v$ in common, and no vertex of $\Sigma_{1} \backslash v$ attaches to $\Sigma_{2} \backslash v$, then

$$
\operatorname{det}(\Sigma, v)=\operatorname{det}\left(\Sigma_{1}, v\right)+\operatorname{det}\left(\Sigma_{2}, v\right)-1 .
$$

Proposition 3.2 ([V1], Prop. 13). If a Coxeter diagram $\Sigma$ is spanned by two disjoint subdiagrams $\Sigma_{1}$ and $\Sigma_{2}$ joined by a unique edge $v_{1} v_{2}$ of weight a, then

$$
\operatorname{det}\left(\Sigma,\left\langle v_{1}, v_{2}\right\rangle\right)=\operatorname{det}\left(\Sigma_{1}, v_{1}\right) \operatorname{det}\left(\Sigma_{2}, v_{2}\right)-a^{2} .
$$

Denote by $\mathcal{L}_{p, q, r}$ a Lannér diagram of order 3 containing subdiagrams of the dihedral groups $G_{2}^{(p)}, G_{2}^{(q)}$ and $G_{2}^{(r)}$. Let $v$ be the vertex of $\mathcal{L}_{p, q, r}$ that does not belong to $G_{2}^{(r)}$, see Fig. 3.1. Denote by $D(p, q, r)$ the local determinant $\operatorname{det}\left(\mathcal{L}_{p, q, r}, v\right)$.

It is easy to check (see e.g. [V1]) that

$$
D(p, q, r)=1-\frac{\cos ^{2}(\pi / p)+\cos ^{2}(\pi / q)+2 \cos (\pi / p) \cos (\pi / q) \cos (\pi / r)}{\sin ^{2}(\pi / r)} .
$$

Notice that $|D(p, q, r)|$ is an increasing function on each of $p, q, r$ tending to infinity while $r$ tends to infinity.

\section{Proof of the Main Theorem}

The plan of the proof is the following. First, we show that there is only a finite number of combinatorial types (or Gale diagrams) of polytopes we are interested in, and we list 


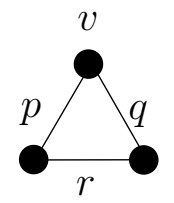

Figure 3.1: Diagram $\mathcal{L}_{p, q, r}$

these Gale diagrams. This was done in Table 3.1. For any Gale diagram from the list we should find all Coxeter polytopes of given combinatorial type. For that, we try to find all Coxeter diagrams with the same structure of Lannér diagrams as the structure of missing faces of the Gale diagram is, and then check the signature. Our task is to be left with finite number of possibilities for each of Gale diagrams, and use a computer after that. Some computations involve a large number of cases, but usually it takes a few minutes of computer's thought. In cases when it is possible to hugely reduce the computations by better estimates we do that, but we follow that by long computations to avoid mistakes.

Lemma 4.1. The following Gale diagrams do not correspond to any hyperbolic Coxeter polytope: $G_{342}, G_{22311}, G_{13131}, G_{352}, G_{424}, G_{31411}$.

Proof. The statement follows from Lemma 3.1. Indeed, the diagram $G_{342}$ contains an arc $J=\lfloor 3,4\rfloor_{1}$. The corresponding Coxeter diagram $\Sigma_{J}$ should be of order 7 , should contain exactly two Lannér diagrams of order 3 and 4 which do not intersect, and should have negative inertia index at most one. Item 1 of Table 3.2 implies that there is no such Coxeter diagram $\Sigma_{J}$. Thus, $G_{342}$ is not a Gale diagram of any hyperbolic Coxeter polytope.

Similarly, Item 1 of Table 3.2 also implies the statement of the lemma for diagrams $G_{352}$ and $G_{424}$. Item 3 implies the statement for $G_{22311}$, Item 11 implies the statement for $G_{13131}$, and Item 5 implies the statement for the diagram $G_{31411}$.

In what follows we check the 14 remaining Gale diagrams case-by-case. We start from larger dimensions.

\subsection{Dimension 7}

In dimension 7 we have only one diagram to consider, namely $G_{13231}$.

Lemma 4.2. There are no compact hyperbolic Coxeter 7 -polytopes with 10 facets.

Proof. Suppose that there exists a compact hyperbolic Coxeter polytope $P$ with Gale diagram $G_{13231}$. This Gale diagram contains an arc $J=\lfloor 3,2,3\rfloor_{2}$. According to Lemma 3.1 (Item 7 of Table 3.2) and Lemma 2.2, the Coxeter diagram $\Sigma$ of $P$ consists of a subdiagram $\Sigma_{J}$ shown in Fig. 4.1, and two nodes $u_{9}, u_{10}$ joined by a dotted edge. By Lemma 2.1, the subdiagrams $\left\langle u_{10}, u_{1}, u_{2}, u_{3}\right\rangle$ and $\left\langle u_{6}, u_{7}, u_{8}, u_{9}\right\rangle$ are Lannér diagrams, and no other 


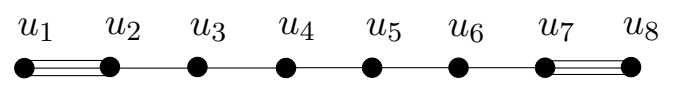

Figure 4.1: A unique diagram $\Sigma_{J}$ for $J=\lfloor 3,2,3\rfloor_{2}$

Lannér subdiagram of $\Sigma$ contains $u_{9}$ or $u_{10}$. In particular, $\Sigma$ does not contain Lannér subdiagrams of order 3.

Consider the diagram $\Sigma^{\prime}=\left\langle\Sigma_{J}, u_{9}\right\rangle$. It is connected and contains neither Lannér diagrams of order 2 or 3 , nor parabolic diagrams. Therefore, $\Sigma^{\prime}$ does not contain neither dotted nor multi-multiple edges. Moreover, by the same reason the node $u_{9}$ may attach to nodes $u_{1}, u_{2}, u_{7}$ and $u_{8}$ by simple edges only. It follows that there are finitely many possibilities for the diagram $\Sigma^{\prime}$. Further, since the diagram $\Sigma^{\prime}$ defines a collection of 9 vectors in 8-dimensional space $\mathbb{R}^{7,1}$, the determinant of $\Sigma^{\prime}$ is equal to zero. A few seconds computer check shows that the only diagrams satisfying conditions listed in this paragraph are the following ones:

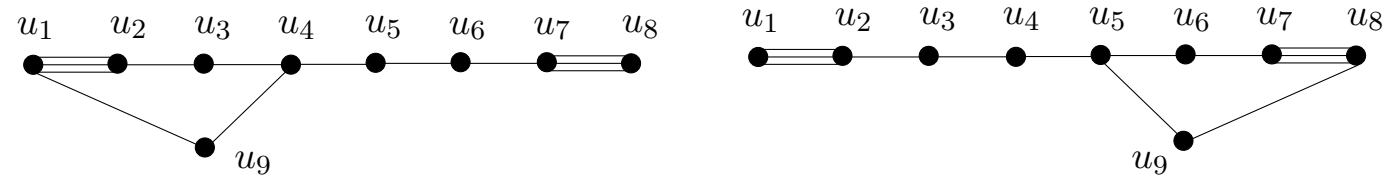

However, the left one contains a Lannér diagram $\left\langle u_{2}, u_{1}, u_{9}, u_{4}, u_{5}\right\rangle$, and the right one contains a Lannér diagram $\left\langle u_{7}, u_{8}, u_{9}, u_{5}, u_{4}\right\rangle$, which is impossible since $u_{9}$ does not belong to any Lannér diagram of order 5.

\subsection{Dimension 6}

In dimension 6 we are left with three diagrams, namely $G_{252}, G_{21411}$, and $G_{12321}$.

Lemma 4.3. There is only one compact hyperbolic Coxeter polytope with Gale diagram $G_{12321}$. Its Coxeter diagram is the lowest one shown in Table 4.9.

Proof. Let $P$ be a compact hyperbolic Coxeter polytope with Gale diagram $G_{12321}$. This Gale diagram contains an arc $J=\lfloor 2,3,2\rfloor_{2}$. According to Lemma 3.1 (Item 6 of Table 3.2) and Lemma 2.2, the Coxeter diagram $\Sigma$ of $P$ consists of a subdiagram $\Sigma_{J}$ shown in Fig. 4.2, and two nodes $u_{8}, u_{9}$ joined by a dotted edge. By Lemma 2.1, the subdiagrams $\left\langle u_{8}, u_{1}, u_{2}\right\rangle$

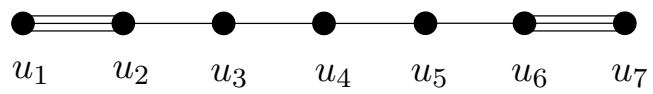

Figure 4.2: A unique diagram $\Sigma_{J}$ for $J=\lfloor 2,3,2\rfloor_{2}$

and $\left\langle u_{6}, u_{7}, u_{9}\right\rangle$ are Lannér diagrams, and no other Lannér subdiagram of $\Sigma$ contains $u_{8}$ or $u_{9}$. So, we need to check possible multiplicities of edges incident to $u_{8}$ and $u_{9}$. 
Consider the diagram $\Sigma^{\prime}=\left\langle\Sigma_{J}, u_{8}\right\rangle$. It is connected, contains neither Lannér diagrams of order 2 nor parabolic diagrams, and contains a unique Lannér diagram of order 3, namely $\left\langle u_{8}, u_{1}, u_{2}\right\rangle$. Therefore, $\Sigma^{\prime}$ does not contain dotted edges, and the only multimultiple edge that may appear should join $u_{8}$ and $u_{1}$.

On the other hand, the signature of $\Sigma_{J}$ is $(6,1)$. This implies that the corresponding vectors in $\mathbb{R}^{6,1}$ form a basis, so the multiplicity of the edge $u_{1} u_{8}$ is completely determined by multiplicities of edges joining $u_{8}$ with the remaining nodes of $\Sigma_{J}$. Since these edges are neither dotted nor multi-multiple, we are left with a finite number of possibilities only. We may reduce further computations observing that $u_{8}$ does not attach to $\left\langle u_{4}, u_{5}, u_{6}, u_{7}\right\rangle$ (since the diagram $\left\langle u_{8}, u_{4}, u_{5}, u_{6}, u_{7}\right\rangle$ should be elliptic), and that multiplicities of edges $u_{8} u_{2}$ and $u_{8} u_{3}$ are at most two and one respectively.

Therefore, we have the following possibilities: $\left[u_{8}, u_{2}\right]=2,3,4$, and, independently, $\left[u_{8}, u_{3}\right]=2,3$. For each of these six cases we should attach the node $u_{8}$ to $u_{1}$ satisfying the condition $\operatorname{det} \Sigma^{\prime}=0$. An explicit calculation shows that there are two diagrams listed below.

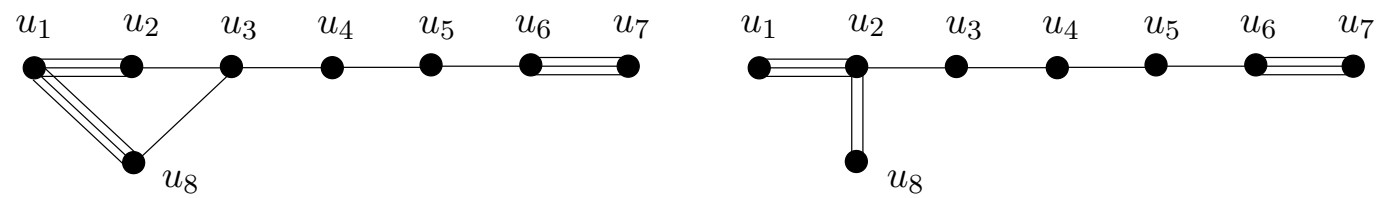

The left one contains a Lannér diagram $\left\langle u_{1}, u_{8}, u_{3}, u_{4}, u_{5}\right\rangle$, which is impossible. At the same time, the right one contains exactly Lannér diagrams prescribed by Gale diagram.

Similarly, the node $u_{9}$ may be attached to $\Sigma_{J}$ in a unique way, i.e. by a unique edge $u_{9} u_{6}$ of multiplicity two. Thus, $\Sigma$ must look like the diagram shown in Fig. 4.3.

Now we write down the determinant of $\Sigma$ as a quadratic polynomial of the weight $d$ of the dotted edge. An easy computation shows that

$$
\operatorname{det} \Sigma=\frac{\sqrt{5}-2}{32}(d-(\sqrt{5}+2))^{2} .
$$

The signature of $\Sigma$ for $d=\sqrt{5}+2$ is equal to $(6,1,2)$, so we obtain that this diagram corresponds to a Coxeter polytope.

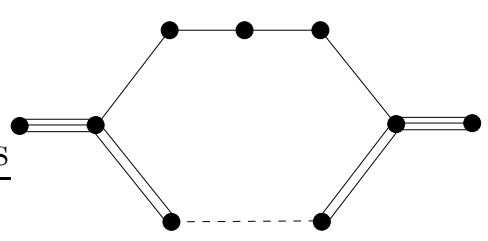

Figure 4.3: Coxeter diagram of a unique Coxeter polytope with Gale diagram $G_{12321}$

Lemma 4.4. There are two compact hyperbolic Coxeter polytopes with Gale diagram $G_{21411}$. Their Coxeter diagrams are shown in the upper row of Table 4.9. 
Proof. Let $P$ be a compact hyperbolic Coxeter polytope with Gale diagram $G_{21411}$. This Gale diagram contains an arc $J=\lfloor 1,4,1\rfloor_{2}$. Hence, the Coxeter diagram $\Sigma$ of $P$ contains a diagram $\Sigma_{J}$ which coincides with one of the three diagrams shown in Item 2 of Table 3.2. Further, $\Sigma$ contains two Lannér diagrams of order 3 , one of which (say, $L$ ) intersects $\Sigma_{J}$. Denote the common node of that Lannér diagram $L$ and $\Sigma_{J}$ by $u_{1}$, the 5 remaining nodes of $\Sigma_{J}$ by $u_{2}, \ldots, u_{6}$ (in a way that $u_{6}$ is marked white in Table 3.2 , i.e. it belongs to only one Lannér diagram of order 5), and denote the two remaining nodes of $L$ by $u_{7}$ and $u_{8}$. Since $L$ is connected, we may assume that $u_{7}$ is joined with $u_{1}$. Notice that $u_{1}$ is also a node marked white in Table 3.2, elsewhere it belongs to at least three Lannér diagrams in $\Sigma$.

Consider the diagram $\Sigma^{\prime}=\left\langle\Sigma_{J}, u_{7}\right\rangle$. It is connected, and all Lannér diagrams contained in $\Sigma^{\prime}$ are contained in $\Sigma_{J}$. In particular, $\Sigma^{\prime}$ does not contain neither dotted nor multi-multiple edges. Hence, we have only finite number of possibilities for $\Sigma^{\prime}$. More precisely, to each of the three diagrams $\Sigma_{J}$ shown in Item 2 of Table 3.2 we must attach a node $u_{7}$ without making new Lannér (or parabolic) diagrams, and all edges must have multiplicities at most 3 . In addition, $u_{7}$ is joined with $u_{1}$. The last condition is restrictive, since we know that $u_{1}$ and $u_{6}$ are the nodes of $\Sigma_{J}$ marked white in Table 3.2. A direct computation (using the technique described in Section 3.2) leads us to the two diagrams $\Sigma_{1}^{\prime}$ and $\Sigma_{2}^{\prime}$ (up to permutation of indices $2,3,4$ and 5 which does not play any role) shown in Fig. 4.4.
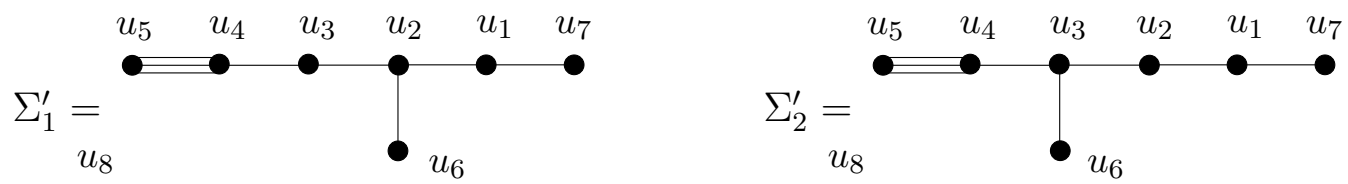

Figure 4.4: Two possibilities for diagram $\Sigma^{\prime}$, see Lemma 4.4

Now consider the diagram $\Sigma^{\prime \prime}=\left\langle\Sigma^{\prime}, u_{8}\right\rangle=\left\langle\Sigma_{J}, u_{7}, u_{8}\right\rangle=\left\langle\Sigma_{J}, L\right\rangle$. As above, $u_{8}$ may attach to $\Sigma_{J}$ by edges of multiplicity at most 3 , so the only multi-multiple edge that may appear in $\Sigma^{\prime \prime}$ is $u_{8} u_{7}$. Since both diagrams $\Sigma_{1}^{\prime}$ and $\Sigma_{2}^{\prime}$ have signature $(6,1)$, the corresponding vectors in $\mathbb{R}^{6,1}$ form a basis, so the multiplicity of the edge $u_{8} u_{7}$ is completely determined by multiplicities of edges joining $u_{8}$ with the remaining nodes of $\Sigma^{\prime}$. Thus, there is a finite number of possibilities for $\Sigma^{\prime \prime}$. To reduce the computations note that $u_{8}$ is not joined with $\left\langle u_{2}, u_{3}, u_{4}, u_{5}\right\rangle$ (since the diagram $\left\langle u_{2}, u_{3}, u_{4}, u_{5}, u_{8}\right\rangle$ must be elliptic). Attaching $u_{8}$ to $\Sigma_{2}^{\prime}$, we do not obtain any diagram with zero determinant and prescribed Lannér diagrams. Attaching $u_{8}$ to $\Sigma_{1}^{\prime}$, we obtain the two diagrams $\Sigma_{1}^{\prime \prime}$ and $\Sigma_{2}^{\prime \prime}$ shown in Fig. 4.5.

The remaining node of $\Sigma$, namely $u_{9}$, is joined with $u_{6}$ by a dotted edge. It is also contained in a Lannér diagram $\left\langle u_{7}, u_{8}, u_{9}\right\rangle$ of order 3 , but no other Lannér diagram contains $u_{9}$. Since $u_{7}$ attaches to $u_{1}$, we see that all edges joining $u_{9}$ with $\Sigma^{\prime} \backslash u_{6}$ are neither dotted nor multi-multiple. On the other hand, for both diagrams $\Sigma_{1}^{\prime \prime}$ and $\Sigma_{2}^{\prime \prime}$, the diagram $\Sigma^{\prime \prime} \backslash u_{6}$ has signature $(6,1)$. Hence, the weight of edge $u_{9} u_{8}$ is completely determined by multiplicities of edges joining $u_{9}$ with the remaining nodes of $\Sigma^{\prime \prime} \backslash u_{6}$, so we are left 

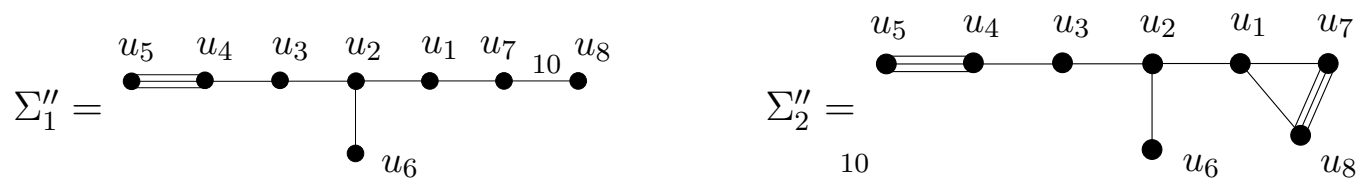

Figure 4.5: Two possibilities for diagram $\Sigma^{\prime \prime}$, see Lemma 4.4

with finitely many possibilities for $\Sigma^{\prime \prime} \backslash u_{6}$. Again, we note that $u_{9}$ is not joined with $\left\langle u_{2}, u_{3}, u_{4}, u_{5}\right\rangle$. Now we attach $u_{9}$ to $u_{1}$ and to $u_{7}$ by edges of multiplicities from 0 (i.e. no edge) to 3 , and then compute the weight of the edge $u_{9} u_{8}$ to $\operatorname{obtain} \operatorname{det}\left(\Sigma \backslash u_{6}\right)=0$. This weight is equal to $\cos \frac{\pi}{m}$ for integer $m$ only in case of the diagrams shown in Fig. 4.6.
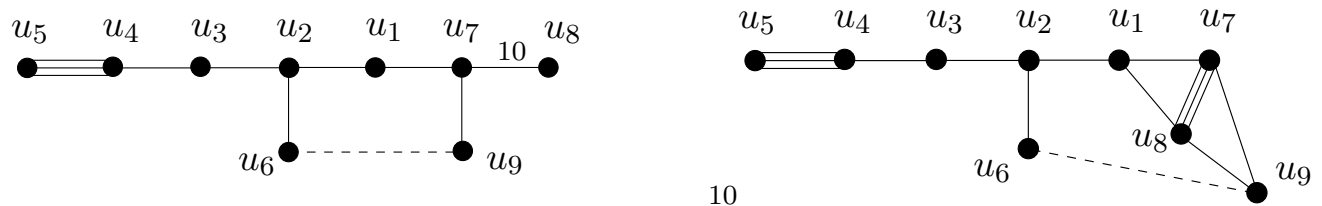

Figure 4.6: Coxeter diagrams of Coxeter polytopes with Gale diagram $G_{21411}$

The last step is to find the weight of the dotted edge $u_{9} u_{6}$ to satisfy the signature condition, i.e. the signature should equal $(6,1,2)$. We write the determinant of $\Sigma$ as a quadratic polynomial of the weight $d$ of the dotted edge, and compute the root. An easy computation shows that for both diagrams the signature of $\Sigma$ for $d=\frac{1+\sqrt{5}}{2}$ is equal to $(6,1,2)$, so we obtain that these two diagrams correspond to Coxeter polytopes. One can note that the right polytope can be obtained by gluing two copies of the left one along the facet corresponding to the node $u_{8}$.

Lemma 4.5. There are no compact hyperbolic Coxeter polytopes with Gale diagram $G_{252}$.

Proof. Suppose that there exists a hyperbolic Coxeter polytope $P$ with Gale diagram $G_{252}$. The Coxeter diagram $\Sigma$ of $P$ contains a Lannér diagram $L_{1}=\left\langle u_{1}, \ldots u_{5}\right\rangle$ of order 5 , and two diagrams of order 2 , denote them $L_{2}=\left\langle u_{6}, u_{8}\right\rangle$ and $L_{3}=\left\langle u_{7}, u_{9}\right\rangle$. The diagram $\left\langle L_{1}, L_{2}\right\rangle$ is connected, otherwise it is superhyperbolic. Thus, we may assume that $u_{6}$ attaches to $L_{1}$. Similarly, we may assume that $u_{7}$ attaches to $L_{1}$.

Therefore, the diagram $\Sigma^{\prime}=\left\langle L_{1}, u_{6}, u_{7}\right\rangle$ consists of a Lannér diagram $L_{1}$ of order 5 and two additional nodes which attach to $L_{1}$, and these nodes are not contained in any Lannér diagram. According to [E1, Lemma 3.8] (see Tabelle 3, page 27, the case $\left|\mathcal{N}_{F}\right|=2$, $\left|\mathcal{L}_{F}\right|=5$ ), $\Sigma^{\prime}$ must coincide with the diagram (up to permutation of indices of nodes of $\left.L_{1}\right)$ shown in Fig. 4.7.

Consider the diagram $\Sigma_{1}^{\prime \prime}=\left\langle\Sigma^{\prime}, u_{8}\right\rangle=\Sigma \backslash u_{9}$. The node $u_{8}$ is joined with $u_{6}$ by a dotted edge. The diagram $\Sigma_{1}^{\prime \prime} \backslash u_{6}$ contains a unique Lannér diagram, $L_{1}$. If $u_{8}$ attaches to $L_{1}$, $\Sigma_{1}^{\prime \prime} \backslash u_{6}$ should coincide with $\Sigma^{\prime}$. Thus, $u_{8}$ does not attach to $\left\langle u_{1}, \ldots, u_{4}\right\rangle$, and $\left[u_{8}, u_{5}\right]=2$ or 3. It is also easy to see that $\left[u_{8}, u_{7}\right] \leq 4$. Since the signature of $\Sigma^{\prime}$ is $(6,1)$, the weight 


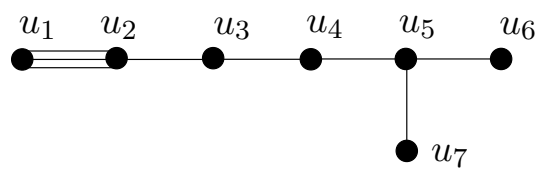

Figure 4.7: The diagram $\Sigma^{\prime}$, see Lemma 4.5

of the edge $u_{8} u_{6}$ is completely determined by multiplicities of edges joining $u_{8}$ with the remaining nodes of $\Sigma^{\prime}$. Hence, we have a finite number of possibilities for $\Sigma_{1}^{\prime \prime}$. To reduce the computations observe that either $\left[u_{5}, u_{8}\right]$ or $\left[u_{7}, u_{8}\right]$ must equal 2 . We are left with only 4 cases: the pair $\left(\left[u_{5}, u_{8}\right],\left[u_{7}, u_{8}\right]\right)$ coincides with one of $(2,2),(2,3),(2,4)$ or $(3,2)$. For each of them we compute the weight of $u_{8} u_{6}$ by solving the equation det $\Sigma_{1}^{\prime \prime}=0$. Each of these equations has one positive and one negative solution, but the positive solution in case of $\left(\left[u_{5}, u_{8}\right],\left[u_{7}, u_{8}\right]\right)=(2,4)$ is less than one, so it cannot be a weight of a dotted edge. Therefore, we have three cases $\left(\left[u_{5}, u_{8}\right],\left[u_{7}, u_{8}\right]\right)=(2,2),(2,3)$ or $(3,2)$, for which

the weight of $u_{8} u_{6}$ is equal to $\frac{\sqrt{2} \sqrt{4+\sqrt{5}}}{\sqrt{11}}, \frac{-3 \sqrt{5}+7+4 \sqrt{10-4 \sqrt{5}}}{\sqrt{-9+5 \sqrt{5}}}$, and $\frac{5+4 \sqrt{5}}{11}$ respectively.

By symmetry, we obtain the same cases for the diagram $\Sigma_{2}^{\prime \prime}=\left\langle\Sigma^{\prime}, u_{9}\right\rangle=\Sigma \backslash u_{8}$, and the same values of the weight of the edge $u_{9} u_{7}$ when $\left(\left[u_{5}, u_{9}\right],\left[u_{6}, u_{9}\right]\right)=(2,2),(2,3)$ and $(3,2)$ respectively. Now, we have only 9 cases to attach nodes $u_{8}$ and $u_{9}$ to $\Sigma^{\prime}$ (in fact, there are only six up to symmetry). For each of these cases we compute the weight of the edge $u_{8} u_{9}$ by solving the equation $\operatorname{det} \Sigma=0$. None of these solutions is equal to $\cos \frac{\pi}{m}$ for integer $m$, which contradicts the fact that the diagram $\left\langle u_{8}, u_{9}\right\rangle$ is elliptic. This contradiction proves the lemma.

\subsection{Dimension 5}

In dimension 5 we must consider six Gale diagrams, namely $G_{242}, G_{323}, G_{21311}, G_{12311}$, $G_{11411}$, and $G_{12221}$.

Lemma 4.6. There is only one compact hyperbolic Coxeter polytope with Gale diagram $G_{12221}$. Its Coxeter diagram is the left one shown in the first row of Table 4.10.

Proof. The proof is similar to the proof of Lemma 4.3. We assume that there exists a hyperbolic Coxeter polytope $P$ with Gale diagram $G_{12221}$. This Gale diagram contains an $\operatorname{arc} J=\lfloor 2,2,2\rfloor_{2}$. According to Lemma 3.1 (Item 10 of Table 3.2) and Lemma 2.2, the Coxeter diagram $\Sigma$ of $P$ consists of the subdiagram $\Sigma_{J}$ shown in Fig. 4.8, and two

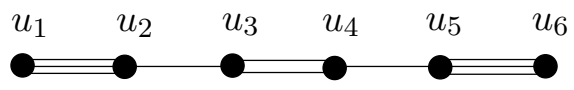

Figure 4.8: A unique diagram $\Sigma_{J}$ for $J=\lfloor 2,2,2\rfloor_{2}$

nodes $u_{7}, u_{8}$ joined by a dotted edge. By Lemma 2.1, the subdiagrams $\left\langle u_{7}, u_{1}, u_{2}\right\rangle$ and 
$\left\langle u_{5}, u_{6}, u_{8}\right\rangle$ are Lannér diagrams, and no other Lannér subdiagram of $\Sigma$ contains $u_{7}$ or $u_{8}$. So, we need to check possible multiplicities of edges incident to $u_{7}$ and $u_{8}$.

Again, we consider the diagram $\Sigma^{\prime}=\left\langle\Sigma_{J}, u_{7}\right\rangle$. It is connected, does not contain dotted edges, and its determinant is equal to zero. Furthermore, observe that $u_{7}$ does not attach to $\left\langle u_{2}, u_{3}, u_{4}, u_{5}\right\rangle$ (since the diagram $\left\langle u_{7}, u_{2}, u_{3}, u_{4}, u_{5}\right\rangle$ should be elliptic), and $u_{7}$ does not attach to $u_{6}$ (since the diagram $\left\langle u_{7}, u_{4}, u_{5}, u_{6}\right\rangle$ should be elliptic). Therefore, $u_{7}$ is joined with $u_{1}$ only. Solving the equation $\operatorname{det} \Sigma^{\prime}=0$, we find that $\left[u_{7}, u_{1}\right]=4$.

By symmetry, we obtain that $u_{8}$ is not joined with $\left\langle u_{1}, u_{2}, u_{3}, u_{4}, u_{5}\right\rangle$, and $\left[u_{8}, u_{6}\right]=4$. Thus, we have the Coxeter diagram $\Sigma$ shown in Fig. 4.9. Assigning the weight $d=$

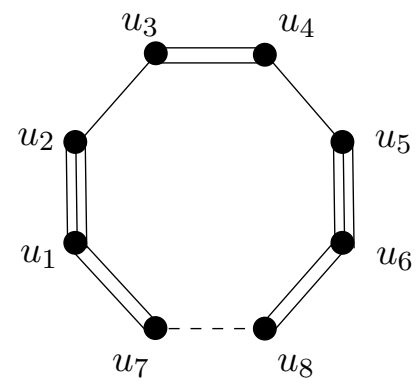

Figure 4.9: Coxeter diagram of a unique Coxeter polytope with Gale diagram $G_{12221}$

$\sqrt{2}(\sqrt{5}+1) / 4$ to the dotted edge, we see that the signature of $\Sigma$ is equal to $(5,1,2)$, so we obtain that this diagram corresponds to a Coxeter polytope.

Before considering the diagram $G_{11411}$, we make a small geometric excursus, the first one in this purely geometric paper.

The combinatorial type of polytope defined by Gale diagram $G_{11411}$ is twice truncated 5 -simplex, i.e. a 5-simplex in which two vertices are truncated by hyperplanes very close to the vertices. If we have such a polytope $P$ with acute angles, it is easy to see that we are always able to truncate the polytope again by two hyperplanes in the following way: we obtain a combinatorially equivalent polytope $P^{\prime}$; the two truncating hyperplanes do not intersect initial truncating hyperplanes and intersect exactly the same facets of $P$ the initial ones do; the two truncating hyperplanes are orthogonal to all facets of $P$ they do intersect.

The difference between polytopes $P$ and $P^{\prime}$ consists of two small polytopes, each of them is combinatorially equivalent to a product of 4-simplex and segment, i.e. each of these polytopes is a simplicial prism. Of course, it is a Coxeter prism, and one of the bases is orthogonal to all facets of the prism it does intersect. All such prisms were classified by Kaplinskaja in $[\mathrm{K}]$. Simplices truncated several times with orthogonality condition described above were classified by Schlettwein in [S]. Twice truncated simplices from the second list are the right ones in rows 1,3 , and 5 of Table 4.10 .

Therefore, to classify all Coxeter polytopes with Gale diagram $G_{11411}$ we only need to do the following. We take a twice truncated simplex from the second list, it has two 
"right" facets, i.e. facets which make only right angles with other facets. Then we find all the prisms that have "right" base congruent to one of "right" facets of the truncated simplex, and glue these prisms to the truncated simplex by "right" facets in all possible ways.

The result is presented in Table 4.10. All polytopes except the left one from the first row have Gale diagram $G_{11411}$. The polytopes from the fifth row are obtained by gluing one prism to the right polytope from this row, the polytopes from the third and fourth rows are obtained by gluing prisms to the right polytope from the third row, and the polytopes from the first and second rows are obtained by gluing prisms to the right polytope from the first row. The number of glued prisms is equal to the number of edges inside the maximal cycle of Coxeter diagram. Hence, we come to the following lemma:

Lemma 4.7. There are 15 compact hyperbolic Coxeter 5-polytopes with 8 facets with Gale diagram $G_{11411}$. Their Coxeter diagrams are shown in Table 4.10.

Proof. In fact, the lemma has been proved above. Here we show how to verify the previous considerations without any geometry and without referring to classifications from $[\mathrm{K}]$ and $[\mathrm{S}]$. Since the procedure is very similar to the proof of Lemma 4.6, we provide only a plan of necessary computations without details.

Let $P$ be a compact hyperbolic Coxeter polytope $P$ with Gale diagram $G_{11411}$. This Gale diagram contains an arc $J=\lfloor 1,4,1\rfloor_{2}$, so the Coxeter diagram $\Sigma$ of $P$ consists of one of the diagrams $\Sigma_{J}$ presented in Item 2 of Table 3.2 and two nodes $u_{7}$ and $u_{8}$ joined by a dotted edge.

Choose one of three diagrams $\Sigma_{J}$. Consider the diagram $\Sigma^{\prime}=\left\langle\Sigma_{J}, u_{7}\right\rangle$. It is connected, contains a unique dotted edge, no multi-multiple edges, and its determinant is equal to zero. So, we are able to find the weight of the dotted edge joining $u_{7}$ with $\Sigma_{J}$ depending on multiplicities of the remaining edges incident to $u_{7}$. The weight of this edge should be greater than one. Of course, we must restrict ourselves to the cases when non-dotted edges incident to $u_{7}$ do not make any new Lannér diagram together with $\Sigma_{J}$. The number of such cases is really small.

Further, we do the same for the diagram $\Sigma^{\prime \prime}=\left\langle\Sigma_{J}, u_{8}\right\rangle$, and we find all possible such diagrams together with the weight of the dotted edge joining $u_{8}$ with $\Sigma_{J}$. Then we are left to determine the weight of the dotted edge $u_{7} u_{8}$ for any pair of diagrams $\Sigma^{\prime}$ and $\Sigma^{\prime \prime}$. It occurs that this weight is always greater than one.

Doing the procedure described above for all the three possible diagrams $\Sigma_{J}$, we obtain the complete list of compact hyperbolic Coxeter 5-polytopes with 8 facets with Gale diagram $G_{11411}$. The computations completely confirm the result of considerations previous to the lemma.

In the remaining part of this section we show that Gale diagrams $G_{242}, G_{323}, G_{21311}$, and $G_{12311}$ do not give rise to any Coxeter polytope.

Lemma 4.8. There are no compact hyperbolic Coxeter polytopes with Gale diagram $G_{12311}$. 
Proof. Suppose that there exists a compact hyperbolic Coxeter polytope $P$ with Gale diagram $G_{12311}$. This Gale diagram contains an arc $J=\lfloor 2,3,1\rfloor_{2}$. According to Lemma 3.1 (Item 9 of Table 3.2) and Lemma 2.2, the Coxeter diagram $\Sigma$ of $P$ consists of one of the nine subdiagrams $\Sigma_{J}$ shown in Table 4.1, and two nodes $u_{7}, u_{8}$ joined by a dotted edge.

Table 4.1: All possible diagrams $\Sigma_{J}$ for $J=\lfloor 2,3,1\rfloor_{2}$
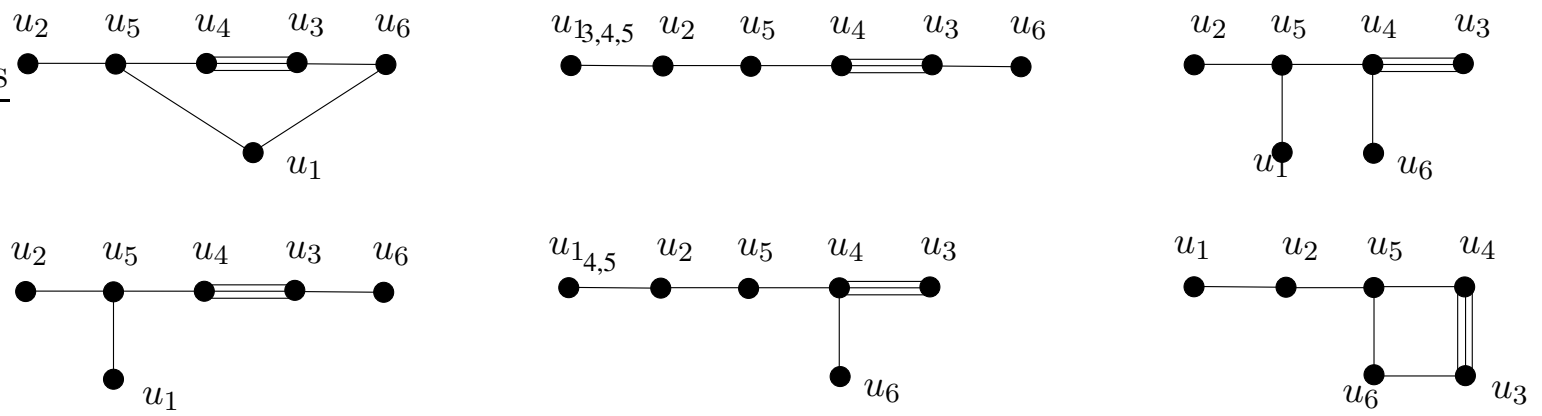

By Lemma 2.1, the subdiagrams $\left\langle u_{7}, u_{1}, u_{2}\right\rangle$ and $\left\langle u_{6}, u_{8}\right\rangle$ are Lannér diagrams, and no other Lannér subdiagram of $\Sigma$ contains $u_{7}$ or $u_{8}$.

Consider the diagram $\Sigma^{\prime}=\left\langle\Sigma_{J}, u_{7}\right\rangle$. It is connected, does not contain dotted edges, and its determinant is equal to zero. Observe that the diagram $\left\langle u_{2}, u_{3}, u_{4}, u_{5}\right\rangle$ is of the type $H_{4}$. Since the diagram $\left\langle u_{7}, u_{2}, u_{3}, u_{4}, u_{5}\right\rangle$ is elliptic, this implies that $u_{7}$ is not joined with $\left\langle u_{2}, u_{3}, u_{4}, u_{5}\right\rangle$. Furthermore, notice that the diagram $\left\langle u_{3}, u_{4}, u_{6}\right\rangle$ is of the type $H_{3}$. Since the diagram $\left\langle u_{7}, u_{3}, u_{4}, u_{6}\right\rangle$ is elliptic, we obtain that $\left[u_{7}, u_{6}\right]=2$ or 3 . Thus, for each of 9 diagrams $\Sigma_{J}$ we have 2 possibilities of attaching $u_{7}$ to $\Sigma_{J} \backslash u_{1}$. Solving the equation $\operatorname{det} \Sigma^{\prime}=0$, we compute the weight of the edge $u_{7} u_{1}$. In all 18 cases the result is not of the form $\cos \frac{\pi}{m}$ for positive integer $m$, which proves the lemma.

Lemma 4.9. There are no compact hyperbolic Coxeter polytopes with Gale diagram $G_{21311}$.

Proof. Suppose that there exists a hyperbolic Coxeter polytope $P$ with Gale diagram $G_{21311}$. This Gale diagram contains an arc $J=\lfloor 1,3,1\rfloor_{2}$. Therefore, the Coxeter diagram $\Sigma$ of $P$ contains one of the five subdiagrams $\Sigma_{J}$, shown in Item 8 of Table 3.2.

On the other hand, $\Sigma$ contains a Lannér diagram $L$ of order 3 intersecting $\Sigma_{J}$. Denote by $u_{1}$ the intersection node of $L$ and $\Sigma_{J}$, and denote by $u_{6}$ and $u_{7}$ the remaining nodes of $L$. Since $L$ is connected, we may assume that $u_{6}$ attaches to $u_{1}$. Denote by $u_{2}$ the node of $\Sigma_{J}$ different from $u_{1}$ and contained in only one Lannér diagram of order 4 , and denote by $u_{3}, u_{4}, u_{5}$ the nodes of $\Sigma_{J}$ contained in two Lannér diagrams of order 4.

Consider the diagram $\Sigma_{0}=\left\langle\Sigma_{J}, u_{6}\right\rangle \backslash u_{2}$. It is connected, has order 5 , and contains a unique Lannér diagram which is of order 4. All such diagrams are listed in [E1, Lemma 3.8] (see the first two rows of Tabelle 3, the case $\left|\mathcal{N}_{F}\right|=1,\left|\mathcal{L}_{F}\right|=4$ ). We have reproduced this list in Table 3.3. 
Consider the diagram $\Sigma_{1}=\left\langle\Sigma_{J}, u_{6}\right\rangle=\left\langle\Sigma_{J}, \Sigma_{0}\right\rangle$. Comparing the lists of possibilities for $\Sigma_{J}$ and $\Sigma_{0}$, it is easy to see that $\Sigma_{1}$ coincides with one of the four diagrams listed in Table 4.2 (up to permutation of indices 3,4 and 5). Now consider the diagram $\Sigma^{\prime}=$

Table 4.2: All possibilities for diagram $\Sigma_{1}$, see Lemma 4.9
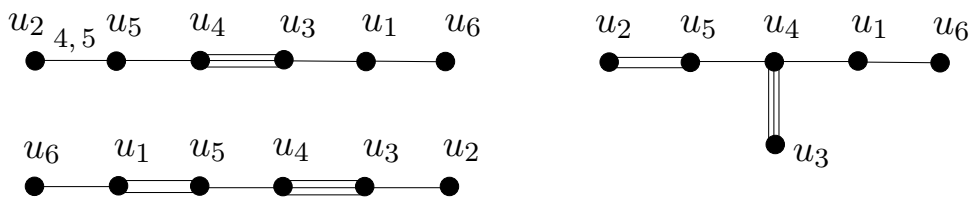

$\left\langle\Sigma_{J}, L\right\rangle=\left\langle\Sigma_{1}, u_{7}\right\rangle$. It is connected, does not contain dotted edges, its determinant is equal to zero, and the only multi-multiple edge may join $u_{7}$ and $u_{6}$. To reduce further computations notice, that the diagram $\left\langle u_{7}, u_{3}, u_{4}, u_{5}\right\rangle$ is elliptic, so $u_{7}$ does not attach to $\left\langle u_{3}, u_{4}\right\rangle$, and may attach to $u_{5}$ by simple edge only. Moreover, since the diagrams $\left\langle u_{7}, u_{2}, u_{4}, u_{5}\right\rangle$ and $\left\langle u_{7}, u_{1}, u_{4}, u_{5}\right\rangle$ are elliptic, $u_{7}$ is not joined with $u_{5}$. Furthermore, since the diagrams $\left\langle u_{7}, u_{1}, u_{4}, u_{5}\right\rangle$ and $\left\langle u_{7}, u_{1}, u_{3}, u_{4}\right\rangle$ are elliptic, $\left[u_{7}, u_{1}\right]=2$ or 3 . Considering elliptic diagrams $\left\langle u_{7}, u_{2}, u_{4}, u_{5}\right\rangle$ and $\left\langle u_{7}, u_{2}, u_{3}, u_{4}\right\rangle$, we obtain that $\left[u_{7}, u_{2}\right]$ is also at most 3. Then for all 4 diagrams $\Sigma_{1}$ and all admissible multiplicities of edges $u_{7} u_{1}$ and $u_{7} u_{2}$ we compute the weight of the edge $u_{7} u_{6}$. We obtain exactly two diagrams $\Sigma^{\prime}$ where this weight is equal to $\cos \frac{\pi}{m}$ for some positive integer $m$, these diagrams are shown in Fig. 4.10. We are left to attach the node $u_{8}$ to $\Sigma^{\prime}$. Consider the diagram $\Sigma^{\prime \prime}=\Sigma \backslash u_{2}$.
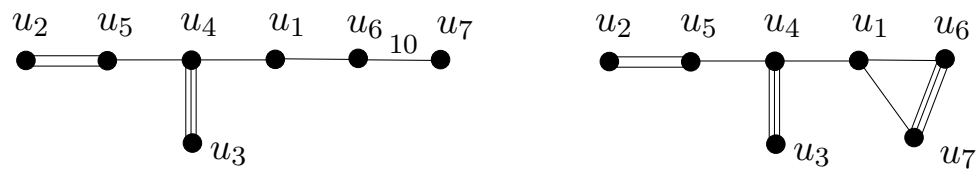

Figure 4.10: All possibilities for diagram $\Sigma^{\prime}$, see Lemma 4.9

As usual, it is connected, does not contain dotted edges, its determinant is equal to zero, and the only multi-multiple edge that may appear is $u_{8} u_{7}$. Furthermore, the diagram $\left\langle u_{3}, u_{4}, u_{1}, u_{6}\right\rangle$ is of the type $H_{4}$, and the diagram $\left\langle u_{8}, u_{3}, u_{4}, u_{1}, u_{6}\right\rangle$ is elliptic. Thus, $u_{8}$ does not attach to $\left\langle u_{3}, u_{4}, u_{1}, u_{6}\right\rangle$. The diagram $\left\langle u_{3}, u_{4}, u_{5}\right\rangle$ is of the type $H_{3}$, and since the diagram $\left\langle u_{8}, u_{3}, u_{4}, u_{5}\right\rangle$ should be elliptic, this implies that $\left[u_{8}, u_{5}\right]=2$ or 3 . Now for both diagrams $\Sigma^{\prime} \backslash u_{2} \subset \Sigma^{\prime \prime}$ we compute the weight of the edge $u_{8} u_{5}$. In all four cases this weight is not equal to $\cos \frac{\pi}{m}$ for any positive integer $m$, that finishes the proof.

Lemma 4.10. There are no compact hyperbolic Coxeter polytope with Gale diagram $G_{323}$.

Proof. Suppose that there exists a hyperbolic Coxeter polytope $P$ with Gale diagram $G_{323}$. The Coxeter diagram $\Sigma$ of $P$ consists of two Lannér diagrams $L_{1}$ and $L_{2}$ of order 3 , and one Lannér diagram $L_{3}$ of order 2. Any two of these Lannér diagrams are joined in $\Sigma$, and any subdiagram of $\Sigma$ not containing one of these three diagrams is elliptic. 
Consider the diagram $\Sigma_{12}=\left\langle L_{1}, L_{2}\right\rangle$. Due to [E2, p. 239, Step 4], we have three cases:

(1) $L_{1}$ and $L_{2}$ are joined by two simple edges having a common vertex, say in $L_{2}$;

(2) $L_{1}$ and $L_{2}$ are joined by a unique double edge;

(3) $L_{1}$ and $L_{2}$ are joined by a unique simple edge.

We fix the following notation: $L_{1}=\left\langle u_{1}, u_{2}, u_{3}\right\rangle, L_{2}=\left\langle u_{4}, u_{5}, u_{6}\right\rangle, L_{3}=\left\langle u_{7}, u_{8}\right\rangle$, the only node of $L_{2}$ joined with $L_{1}$ is $u_{4} ; u_{4}$ is joined with $u_{3}$ and, in case (1), with $u_{1}$. We may assume also that $u_{7}$ attaches to $L_{1}, u_{4}$ is joined to $u_{5}$ in $L_{2}$, and $u_{2}$ is joined to $u_{3}$ in $L_{1}$. Case (1). Since the diagrams $\left\langle u_{2}, u_{1}, u_{4}\right\rangle$ and $\left\langle u_{2}, u_{3}, u_{4}\right\rangle$ are elliptic, $\left[u_{2}, u_{1}\right]$ and $\left[u_{2}, u_{3}\right]$ do not exceed 5 . On the other hand, $\left\langle u_{1}, u_{2}, u_{3}\right\rangle=L_{1}$ is a Lannér diagram, so we may assume that $\left[u_{2}, u_{1}\right]=5$, and $\left[u_{2}, u_{3}\right]=4$ or 5 . Now attach $u_{7}$ to $L_{1}$. If $u_{7}$ is joined with $u_{1}$ or $u_{2}$, then the diagram $\left\langle u_{2}, u_{1}, u_{4}\right\rangle$ is not elliptic, and if $u_{7}$ is joined with $u_{3}$, then the diagram $\left\langle u_{2}, u_{3}, u_{4}\right\rangle$ is not elliptic, which contradicts Lemma 2.1.

Case (2). It is clear that $\left[u_{2}, u_{3}\right]=\left[u_{4}, u_{5}\right]=3$, and $u_{7}$ cannot be attached to $u_{3}$. Thus, $u_{7}$ is joined with $u_{1}$ or $u_{2}$, which implies that $\left[u_{2}, u_{1}\right] \leq 5$. Therefore, $\left[u_{1}, u_{3}\right]=3$. So, the diagrams $\left\langle u_{1}, u_{3}, u_{4}, u_{5}\right\rangle$ and $\left\langle u_{2}, u_{3}, u_{4}, u_{5}\right\rangle$ are of the type $F_{4}$. Therefore, if $u_{7}$ attaches $u_{1}$, then the diagram $\left\langle u_{7}, u_{1}, u_{3}, u_{4}, u_{5}\right\rangle$ is not elliptic, and if $u_{7}$ is joined with $u_{2}$, then the diagram $\left\langle u_{7}, u_{2}, u_{3}, u_{4}, u_{5}\right\rangle$ is not elliptic.

Case (3). The signature of $\Sigma_{12}$ is either $(5,1)$ or $(4,1,1)$. Thus, $\operatorname{det} \Sigma_{12} \leq 0$. By Prop. 3.2, $\operatorname{det}\left(L_{1}, u_{3}\right) \operatorname{det}\left(L_{2}, u_{4}\right) \leq \frac{1}{4}$. We may assume that $\left|\operatorname{det}\left(L_{1}, u_{3}\right)\right| \leq\left|\operatorname{det}\left(L_{2}, u_{4}\right)\right|$, in particular, $\left|\operatorname{det}\left(L_{1}, u_{3}\right)\right| \leq \frac{1}{2}$. By [E2, Table 2], there are only 6 possibilities for $\left\langle L_{1}, u_{4}\right\rangle$, we list them in Table 4.3.

Table 4.3: All possibilities for diagram $\left\langle L_{1}, u_{4}\right\rangle$, see Case (3) of Lemma 4.10
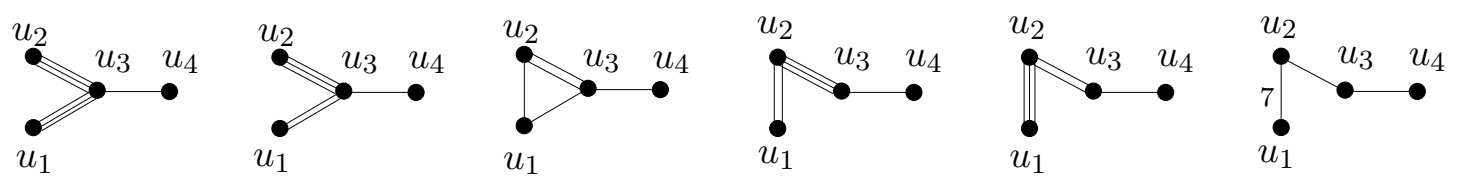

For any of these six diagrams $\left|\operatorname{det}\left(L_{1}, u_{3}\right)\right| \geq \frac{\sqrt{5}-1}{8}$. Thus, $\left|\operatorname{det}\left(L_{2}, u_{4}\right)\right| \leq \frac{1}{4} \frac{8}{\sqrt{5}-1}=\frac{2}{\sqrt{5}-1}$. Notice that since the diagrams $\left\langle u_{3}, u_{4}, u_{5}\right\rangle$ and $\left\langle u_{3}, u_{4}, u_{6}\right\rangle$ are elliptic, $\left[u_{4}, u_{5}\right]$ and $\left[u_{4}, u_{6}\right]$ do not exceed 5 . Now, since the local determinant is an increasing function of multiplicities of the edges, it is not difficult to list all Lannér diagrams $L_{2}=\left\langle u_{4}, u_{5}, u_{6}\right\rangle$, such that $\left[u_{4}, u_{5}\right],\left[u_{4}, u_{6}\right] \leq 5$, and $\left|\operatorname{det}\left(L_{2}, u_{4}\right)\right| \leq \frac{2}{\sqrt{5}-1}$. This list contains 17 diagrams only.

Then, from $6 \cdot 17=102$ pairs $\left(L_{1}, L_{2}\right)$ we list all pairs with $\operatorname{det}\left(L_{1}, u_{3}\right) \operatorname{det}\left(L_{2}, u_{4}\right) \leq \frac{1}{4}$. Each of these pairs corresponds to a diagram $\Sigma_{12}$. After that, we attach to all diagrams $\Sigma_{12}$ a node $u_{7}$ in the following way: $u_{7}$ is joined with $L_{1}$ (and may be joined with $L_{2}$, too), and it does not produce any new Lannér or parabolic diagram. It occurs that none of obtained diagrams $\left\langle\Sigma_{12}, u_{7}\right\rangle$ has zero determinant.

Lemma 4.11. There are no compact hyperbolic Coxeter polytopes with Gale diagram $G_{242}$. 
Proof. Suppose that there exists a hyperbolic Coxeter polytope $P$ with Gale diagram $G_{242}$. The Coxeter diagram $\Sigma$ of $P$ consists of one Lannér diagram $L_{1}$ of order 4 , and two Lannér diagrams $L_{2}$ and $L_{3}$ of order 2. Any two of these Lannér diagrams are joined in $\Sigma$, and any subdiagram of $\Sigma$ not containing one of these three diagrams is elliptic.

We fix the following notation: $L_{1}=\left\langle u_{1}, u_{2}, u_{3}, u_{4}\right\rangle, L_{2}=\left\langle u_{5}, u_{7}\right\rangle, L_{3}=\left\langle u_{6}, u_{8}\right\rangle, u_{5}$ and $u_{6}$ attach to $L_{1}$.

Consider the diagram $\Sigma_{0}=\left\langle L_{1}, u_{5}, u_{6}\right\rangle$. It is connected, has order 6 , and contains a unique Lannér diagram which is of order 4 . All such diagrams are listed in [E1, Lemma 3.8] (see Tabelle 3 , the first two rows of page 27 , the case $\left|\mathcal{N}_{F}\right|=2,\left|\mathcal{L}_{F}\right|=4$ ). We have reproduced this list in Table 3.4. The list contains five diagrams, but we are interested in four of them: in the fifth one only one of two additional nodes attaches to the Lannér diagram. We list these four possibilities for $\Sigma_{0}$ in Table 4.4 .

Table 4.4: All possibilities for diagram $\Sigma_{0}$, see Lemma 4.11
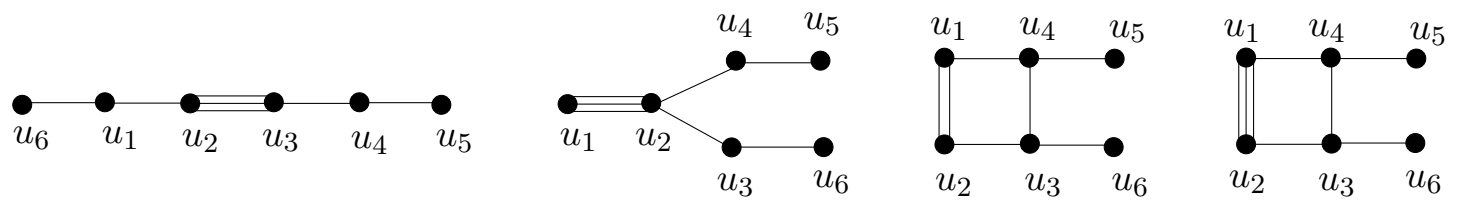

Now consider the diagram $\Sigma^{\prime}=\left\langle\Sigma_{0}, u_{7}\right\rangle$. It contains a unique dotted edge $u_{5} u_{7}$. Since the diagram $\left\langle u_{7}, u_{1}, u_{2}, u_{3}, u_{6}\right\rangle$ is elliptic and the diagram $\left\langle u_{1}, u_{2}, u_{3}, u_{6}\right\rangle$ is of the type $H_{4}$ or $B_{4}, u_{7}$ is not joined with $\left\langle u_{1}, u_{2}, u_{3}\right\rangle$, and it may attach to $u_{6}$ if $\left[u_{1}, u_{2}\right]=4$ only. It is easy to see that $\left[u_{7}, u_{4}\right]=2$ or 3 in all four cases. We obtain 9 possibilities for attaching $u_{7}$ to $\Sigma_{0} \backslash u_{5}$. For each of them we compute the weight of the edge $u_{5} u_{7}$.

By symmetry, we may list all 9 possibilities for the diagram $\Sigma^{\prime \prime}=\left\langle\Sigma_{0}, u_{8}\right\rangle$. Now we are left to compute the weight of the edge $u_{7} u_{8}$ in $\Sigma$. Diagrams $\Sigma_{0}$ with $\left[u_{1}, u_{2}\right]=5$ produce three possible diagrams $\Sigma$ each, and the diagram $\Sigma_{0}$ with $\left[u_{1}, u_{2}\right]=4$ produces six possible diagrams $\Sigma$ (we respect symmetry). In all these 15 cases the weight of the edge $u_{7} u_{8}$ is not of the form $\cos \frac{\pi}{m}$ for positive integer $m$.

\subsection{Dimension 4}

In dimension 4 we must consider four Gale diagrams, namely $G_{232}, G_{11311}, G_{21112}$, and $G_{12121}$. Three of them, i.e. $G_{232}, G_{11311}$, and $G_{12121}$, give rise to Coxeter polytopes.

Lemma 4.12. There are exactly three compact hyperbolic Coxeter polytopes with Gale diagram $G_{232}$. Their Coxeter diagrams are shown in the third row of the second part of Table 4.11.

Proof. Let $P$ be a compact hyperbolic Coxeter polytope with Gale diagram $G_{232}$. The Coxeter diagram $\Sigma$ of $P$ consists of one Lannér diagram $L_{1}$ of order 3 , and two Lannér 
diagrams $L_{2}$ and $L_{3}$ of order 2. Any two of these Lannér diagrams are joined in $\Sigma$, and any subdiagram of $\Sigma$ containing none of these three diagrams is elliptic.

On the first sight, the considerations may repeat ones from the proof of Lemma 4.11. However, there is a small difference: the number of Lannér diagrams of order 3 is infinite. Thus, at first we must bound the multiplicities of the edges of the Lannér diagram of order 3 .

We fix the following notation: $L_{1}=\left\langle u_{1}, u_{2}, u_{3}\right\rangle, L_{2}=\left\langle u_{5}, u_{6}\right\rangle, L_{3}=\left\langle u_{4}, u_{7}\right\rangle, u_{4}$ and $u_{5}$ attach to $L_{1}$. We may also assume that $u_{4}$ attaches to $u_{3}$.

Since the diagrams $\left\langle u_{1}, u_{3}, u_{4}\right\rangle$ and $\left\langle u_{2}, u_{3}, u_{4}\right\rangle$ should be elliptic, the edges $u_{3} u_{1}$ and $u_{3} u_{2}$ are not multi-multiple. We consider two cases: $u_{1}$ or $u_{2}$ is either joined with $\left\langle u_{4}, u_{5}, u_{6}, u_{7}\right\rangle$ or not.

Case 1: $u_{1}$ and $u_{2}$ are not joined with $\left\langle u_{4}, u_{5}, u_{6}, u_{7}\right\rangle$. In particular, this is true if the edge $u_{1} u_{2}$ is multi-multiple. Then $u_{5}$ attaches to $u_{3}$. Since the diagrams $\left\langle u_{1}, u_{3}, u_{4}, u_{5}\right\rangle$ and $\left\langle u_{2}, u_{3}, u_{4}, u_{5}\right\rangle$ are elliptic, $\left[u_{3}, u_{1}\right]$ and $\left[u_{3}, u_{2}\right]$ do not exceed $3,\left[u_{3}, u_{4}\right]=\left[u_{3}, u_{5}\right]=3$, and $\left[u_{4}, u_{5}\right]=2$. We may assume that $\left[u_{3}, u_{1}\right]=3$, and $\left[u_{3}, u_{2}\right]=2$ or 3 .

Consider the diagram $\Sigma^{\prime}=\left\langle L_{1}, L_{2}, u_{4}\right\rangle=\Sigma \backslash u_{7}$. We know that $u_{6}$ is joined with $u_{5}$ by a dotted edge, and $u_{6}$ does not attach to $u_{1}$ and $u_{2}$. Furthermore, since the diagram $\left\langle u_{1}, u_{3}, u_{4}, u_{6}\right\rangle$ is elliptic, $\left[u_{6}, u_{3}\right] \leq 3$ and $\left[u_{6}, u_{4}\right] \leq 4$. By the same reason, either $\left[u_{6}, u_{3}\right]$ or $\left[u_{6}, u_{4}\right]$ is equal to 2 . Thus, we have four possibilities to attach $u_{6}$ to $u_{3}$ and $u_{4}$.

Denote by $d$ the weight of the dotted edge $u_{5} u_{6}$, and compute the local determinant $\operatorname{det}\left(\left\langle u_{3}, u_{4}, u_{5}, u_{6}\right\rangle, u_{3}\right)$ for all four diagrams $\left\langle u_{3}, u_{4}, u_{5}, u_{6}\right\rangle$ as a function of $d$.

Case 1.1: $\left[u_{6}, u_{4}\right] \neq 2$. In this case $\operatorname{det}\left(\left\langle u_{3}, u_{4}, u_{5}, u_{6}\right\rangle, u_{3}\right)$ equals either $\frac{12 d^{2}+4 d-5}{4\left(4 d^{2}-3\right)}$ (when $\left.\left[u_{6}, u_{4}\right]=3\right)$ or $\frac{6 d^{2}+2 \sqrt{2} d-1}{4\left(2 d^{2}-1\right)}\left(\right.$ when $\left.\left[u_{6}, u_{4}\right]=4\right)$. Both expressions decrease in the ray $[1, \infty)$, so the maximal values are $11 / 4$ and $(5+2 \sqrt{2}) / 4$ respectively. Now recall that $\operatorname{det} \Sigma^{\prime}=0$, so by Prop. 3.1 we have $\operatorname{det}\left(L_{1}, u_{3}\right)=1-\operatorname{det}\left(\left\langle u_{3}, u_{4}, u_{5}, u_{6}\right\rangle, u_{3}\right)$. Therefore, $\left|\operatorname{det}\left(L_{1}, u_{3}\right)\right|$ is bounded from above by $7 / 4$ or $(1+2 \sqrt{2}) / 4$ if $\left[u_{6}, u_{4}\right]=3$ or $\left[u_{6}, u_{4}\right]=4$ respectively. Since $\left|\operatorname{det}\left(L_{1}, u_{3}\right)\right|$ is an increasing function on $\left[u_{1}, u_{2}\right]$, an easy check shows that $\left[u_{1}, u_{2}\right]$ is bounded by 10 or 8 respectively. So, in both cases we have finitely many possibilities for $L_{1}$.

Further considerations follow ones from Lemma 4.11. We list all possible $\Sigma^{\prime}$ together with the weight of the dotted edge $u_{5} u_{6}$ (which may be computed from the equation $\operatorname{det} \Sigma^{\prime}=0$ ), then we list all possible diagrams $\Sigma^{\prime \prime}=\left\langle L_{1}, L_{3}, u_{5}\right\rangle=\Sigma \backslash u_{6}$ in a similar way. After that for all pairs $\left(\Sigma^{\prime}, \Sigma^{\prime \prime}\right)$ (with the same $L_{1}$ ) we compute the weight of the edge $u_{6} u_{7}$. It occurs that in all cases the weight is not of the form $\cos \frac{\pi}{m}$ for positive integer $m$.

Case 1.2: $\left[u_{6}, u_{4}\right]=2$. In this case $\operatorname{det}\left(\left\langle u_{3}, u_{4}, u_{5}, u_{6}\right\rangle, u_{3}\right)$ equals either $\frac{3 d^{2}-2}{4\left(d^{2}-1\right)}$ (when $\left.\left[u_{6}, u_{3}\right]=2\right)$ or $\frac{3 d-1}{4(d-1)}\left(\right.$ when $\left.\left[u_{6}, u_{3}\right]=3\right)$. These tend to $\infty$ when $d$ tends to 1 , so we do not obtain any bound for $\left[u_{1}, u_{2}\right]$.

Let $m_{12}=\left[u_{1}, u_{2}\right], m_{23}=\left[u_{2}, u_{3}\right]$, and let $m_{36}=\left[u_{3}, u_{6}\right]$. Notice that $m_{23}, m_{36}=2$ or 3. Define also $c_{12}=\cos \left(\pi / m_{12}\right)$. We compute the weight of the edge $u_{5} u_{6}$ as a function $d\left(m_{12}, m_{23}, m_{36}\right)$ of $m_{12}, m_{23}$ and $m_{36}$. Solving the equation $\operatorname{det} \Sigma^{\prime}=0$, we see that 


$$
\begin{gathered}
d\left(m_{12}, 2,2\right)=\sqrt{\frac{2 c_{12}^{2}-1}{2 c_{12}^{2}-2} ;} \quad d\left(m_{12}, 3,2\right)=\sqrt{\frac{2 c_{12}}{3 c_{12}-1}} ; \\
d\left(m_{12}, 2,3\right)=\frac{c_{12}^{2}}{3 c_{12}^{2}-2} ; \quad d\left(m_{12}, 3,3\right)=\frac{c_{12}+1}{3 c_{12}-1} .
\end{gathered}
$$

Consider the diagram $\Sigma$. According to Case 1.1, we may assume that $\left[u_{5}, u_{7}\right]=2$. Since $L_{2}$ and $L_{3}$ are joined in $\Sigma,\left[u_{6}, u_{7}\right] \neq 2$. On the other hand, the diagram $\left\langle u_{3}, u_{6}, u_{7}\right\rangle$ is elliptic. Thus, either $\left[u_{3}, u_{6}\right]$ or $\left[u_{3}, u_{7}\right]$ equals 2 . By symmetry, we may assume that $\left[u_{3}, u_{7}\right]=2$. We also know how the weight of the edge $u_{4} u_{7}$ depends on $m_{12}$ and $m_{23}$.

Now we are able to compute the weight $w\left(m_{12}, m_{23}, m_{36}\right)$ of the dotted edge $u_{4} u_{7}$ as a function of $m_{12}, m_{23}$ and $m_{36}$. For that we simply solve the equation det $\Sigma=0$. Notice that since $L_{1}$ is a Lannér diagram, $m_{12} \geq 7$ when $m_{23}=2$, and $m_{12} \geq 4$ when $m_{23}=3$. We obtain:

- $w\left(m_{12}, 2,2\right)=\frac{1-c_{12}^{2}}{3 c_{12}^{2}-2}$ is a decreasing function of $m_{12}$ as $m_{12} \geq 7$, and $w(7,2,2)<1 / 2$;

- $w\left(m_{12}, 2,3\right)=\frac{2\left(1-c_{12}^{2}\right) \sqrt{2 c_{12}^{2}}}{\left(3 c_{12}^{2}-2\right)^{3 / 2}}$ is a decreasing function of $m_{12}$ as $m_{12} \geq 7, w(9,2,3)<$ $1 / 2$, and $w\left(m_{12}, 2,3\right) \neq \cos (\pi / m)$ when $m_{12}=7$ or 8 ;

- $w\left(m_{12}, 3,2\right)=\frac{1-c_{12}}{3 c_{12}-1}$ is a decreasing function of $m_{12}$ as $m_{12} \geq 4$, and $w(4,2,2)<1 / 2$; - $w\left(m_{12}, 3,3\right)=\frac{2\left(1-c_{12}\right) \sqrt{2 c_{12}}}{\left(3 c_{12}-1\right)^{3 / 2}}$ is a decreasing function of $m_{12}$ as $m_{12} \geq 4, w(5,3,3)<$ $1 / 2$, and $w(4,3,3) \neq \cos (\pi / m)$.

This finishes considerations of Case 1.

Case 2: either $u_{1}$ or $u_{2}$ is joined with $\left\langle u_{4}, u_{5}, u_{6}, u_{7}\right\rangle$. In particular, this implies that $L_{1}$ contains no multi-multiple edges, so we deal with a finite number of possibilities for $L_{1}$ only. This list contains 11 Lannér diagrams of order 3 . Using that list, it is not too difficult to list all the diagrams $\Sigma_{0}=\left\langle L_{1}, u_{4}, u_{5}\right\rangle$. This list contains 19 diagrams, we present them in Table 4.5. Now we follow the proof of Lemma 4.11. Choose one of 19

Table 4.5: All possibilities for diagram $\Sigma_{0}$, see Case 2 of Lemma 4.12
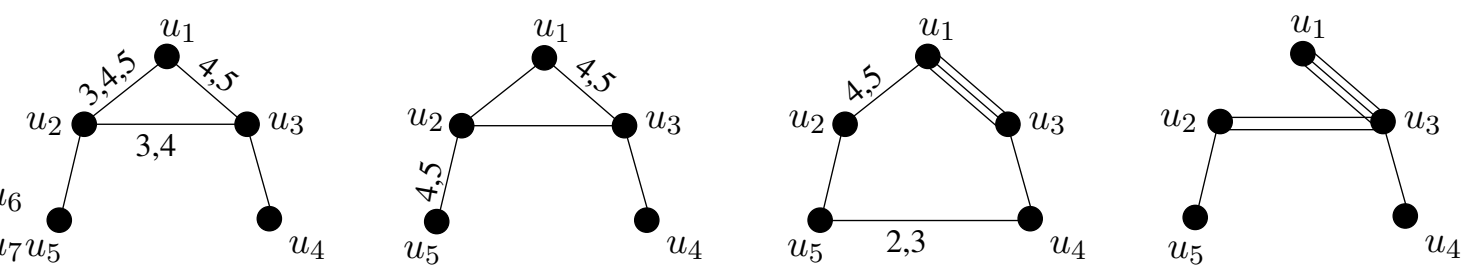

diagrams $\Sigma_{0}$, and consider the diagram $\Sigma^{\prime}=\left\langle\Sigma_{0}, u_{6}\right\rangle$. It contains a unique dotted edge 
$u_{5} u_{6}$, and that is the only Lannér diagram in $\Sigma^{\prime}$ containing $u_{6}$. We have a finite number of possibilities to attach $u_{6}$ to $\Sigma_{0} \backslash u_{5}$. For each of them we compute the weight of the edge $u_{5} u_{6}$.

Similarly, we list all possibilities for the diagram $\Sigma^{\prime \prime}=\left\langle\Sigma_{0}, u_{7}\right\rangle$. Now we are left to compute the weight of the edge $u_{6} u_{7}$ in $\Sigma$. A computation shows that the weight is of the form $\cos \frac{\pi}{m}$ only for the diagrams listed in Table 4.6. To verify that these diagrams

Table 4.6: Coxeter diagrams of Coxeter polytopes with Gale diagram $G_{232}$
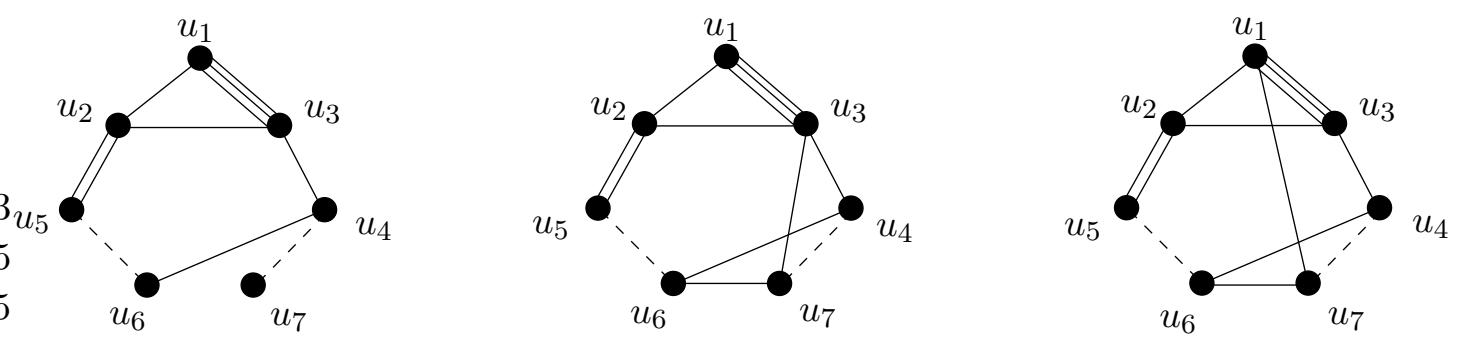

correspond to polytopes, we need to assign weights to the dotted edges. We assign a weight $\sqrt{2} \frac{\sqrt{5}+1}{4}$ to all edges $u_{5} u_{6}$, and weights $\frac{\sqrt{15(5+\sqrt{5})}}{10}, \frac{5+3 \sqrt{5}}{10}$ and $\frac{3+\sqrt{5}}{4}$ to the edge $u_{4} u_{7}$ on the left, middle and right diagrams respectively. A direct calculation shows that the diagrams have signature $(4,1,2)$.

Lemma 4.13. There are 29 compact hyperbolic Coxeter polytopes with Gale diagram $G_{11311}$. Their Coxeter diagrams are shown in the first part of Table 4.11 and in the first three rows of the second part of the same table.

Proof. The proof is identical to one which concerns the diagram $G_{1} 1411$ (see Lemma 4.7). The combinatorial type of polytope defined by Gale diagram $G_{11311}$ is twice truncated 4-simplex. Any such Coxeter polytope may be obtained by gluing one or two prisms to a twice truncated 4-simplex with orthogonality conditions described before Lemma 4.7. Such simplices were classified by Schlettwein in [S], they appear as right ones in rows 1, 2, and 4 of the first part of Table 4.11, and in rows 1 and 2 of the second part. The prisms were classified by Kaplinskaja in $[\mathrm{K}]$.

For each twice truncated simplex from the list of Schlettwein we find all the prisms that have "right" base congruent to one of "right" facets of the truncated simplex, and glue these prisms to the truncated simplex. The result is presented in Table 4.11.

The verification of the result above by computations is completely identical to the proof of Lemma 4.7. We only need to replace an arc $J=\lfloor 1,4,1\rfloor_{2}$ from $G_{1} 1411$ by an arc $J=\lfloor 1,3,1\rfloor_{2}$, and refer to Item 8 of Table 3.2 instead of Item 2 .

Lemma 4.14. There are no compact hyperbolic Coxeter polytopes with Gale diagram $G_{21112}$. 
Proof. Suppose that there exists a hyperbolic Coxeter polytope $P$ with Gale diagram $G_{21112}$. The Coxeter diagram $\Sigma$ of $P$ consists of one Lannér diagram $L_{1}=\left\langle u_{1}, u_{2}, u_{3}, u_{4}\right\rangle$ of order 4 , two Lannér diagrams $L_{2}=\left\langle u_{6}, u_{1}, u_{2}\right\rangle$ and $L_{3}=\left\langle u_{3}, u_{4}, u_{5}\right\rangle$ of order 3 , and two Lannér diagrams $\left\langle u_{6}, u_{7}\right\rangle$ and $\left\langle u_{7}, u_{5}\right\rangle$ of order 2.

Consider the diagram $\Sigma^{\prime}=\left\langle L_{1}, L_{2}, L_{3}\right\rangle=\Sigma \backslash u_{7}$. It is connected, has order 6 , and contains no dotted edges. We may also assume that $u_{5}$ attaches to $u_{4}$. Clearly, any multimultiple edge that may appear in $\Sigma^{\prime}$ belongs to $L_{2}$ or $L_{3}$ and does not belong to $L_{1}$. We consider two cases: either $\Sigma^{\prime}$ contains multi-multiple edges or not.

Suppose that $\Sigma^{\prime}$ contains no multi-multiple edges. Then we have 9 possibilities for $L_{2}$, and 9 possibilities for $L_{3}$. For each of 81 pairs (or 45 in view of symmetry) we join nodes of $L_{2}$ with nodes of $L_{3}$ in all possible ways (9 edges, 4 possibilities for each of them, from empty to triple one). We are looking for diagrams satisfying the following conditions: the determinant should vanish, there are no parabolic subdiagrams, and the diagram contains a unique new Lannér diagram, which has order 4 . A computer check (which takes about 10 hours of computer thought) shows that only 39 obtained diagrams have zero determinant, and only 11 of them contain Lannér diagrams of order 4 . However, each of them contains some new Lannér diagram of order 3. Therefore, none of them may be considered as $\Sigma^{\prime}$.

Now suppose that $\Sigma^{\prime}$ contains at least one multi-multiple edge. We may assume that $u_{4} u_{5}$ is multi-multiple. In this case $u_{4}$ must be a leaf of $L_{1}$, i.e. it should have valency one in $L_{1}$. Indeed, if $u_{4}$ is joined with two vertices $v, w \in L_{1}$, then both diagrams $\left\langle u_{5}, u_{4}, v\right\rangle$ and $\left\langle u_{5}, u_{4}, w\right\rangle$ are not elliptic, which is impossible. Thus, $L_{1}$ is not a cycle, so we have 4 possibilities for $L_{1}$ only (see Table 2.2 ). In Table 4.7 we list all possible diagrams $L_{1}$ together with all possible numerations of nodes. A numeration should satisfy the following properties: $u_{4}$ is a leaf, and $u_{3}$ is a unique neighbor of $u_{4}$. We consider numerations up to interchange of $u_{1}$ and $u_{2}$.

Table 4.7: Numberings of vertices of Lannér diagrams of order 4 without cycles

(1)
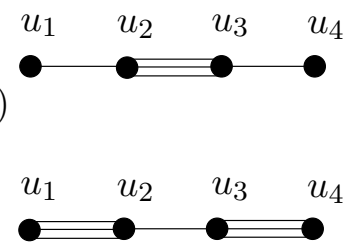

(3) (2a)
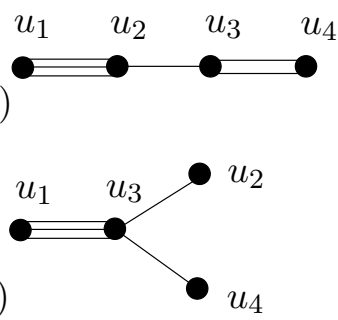

$(2 b)$

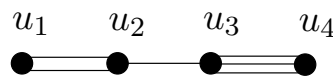

$(4 \mathrm{~b})$

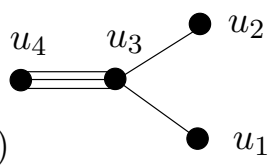

Consider 6 diagrams case-by-case. For all of them we claim that $u_{5}$ and $u_{4}$ do not attach to $L_{2}=\left\langle u_{1}, u_{2}, u_{6}\right\rangle$ : this is because the edge $u_{4} u_{5}$ is multi-multiple.

Diagram (1). Since the diagram $\left\langle u_{1}, u_{2}, u_{3}, u_{5}\right\rangle$ is elliptic, $u_{5}$ is not joined with $u_{3}$. Furthermore, since the diagram $\left\langle u_{6}, u_{2}, u_{3}, u_{4}\right\rangle$ is elliptic, $u_{6}$ is not joined with $\left\langle u_{2}, u_{3}\right\rangle$. Therefore, $\left[u_{6}, u_{2}\right]=2$, so $\left[u_{6}, u_{1}\right] \geq 7$. Applying Prop. 3.2, we see that $\operatorname{det}\left(L_{2}, u_{2}\right) \operatorname{det}\left(L_{3}, u_{3}\right)=$ $\cos ^{2}(\pi / 5)$. An easy calculation shows that the inequality $\left[u_{6}, u_{1}\right] \geq 7$ implies that 
$\left[u_{4}, u_{5}\right] \leq 10$. By symmetry, $\left[u_{6}, u_{1}\right] \leq 10$, too. We are left with a finite (and very small) number of possibilities for $\Sigma^{\prime}$. For none of them $\operatorname{det} \Sigma^{\prime}=0$.

Diagrams (2a), (2b) and (3). Since the diagram $\left\langle u_{1}, u_{2}, u_{3}, u_{5}\right\rangle$ is elliptic, $\left[u_{3}, u_{5}\right] \leq 3$. Since the diagram $\left\langle u_{6}, u_{2}, u_{3}, u_{4}\right\rangle$ is elliptic, $u_{6}$ is not joined with $u_{3}$, and $\left[u_{6}, u_{2}\right] \leq 3$, so $\left[u_{6}, u_{1}\right] \geq 3$. Applying Prop. 3.2, we have $\operatorname{det}\left(L_{2}, u_{2}\right) \operatorname{det}\left(L_{3}, u_{3}\right)=1 / 4$. By assumption, $\left[u_{4}, u_{5}\right] \geq 6$, which implies the inequality $\left|\operatorname{det}\left(L_{3}, u_{3}\right)\right| \geq|D(2,4,6)|=1$. Thus, $\left|\operatorname{det}\left(L_{2}, u_{2}\right)\right| \leq 1 / 4$. But since $\left[u_{1}, u_{2}\right] \geq 4$ and $\left[u_{6}, u_{2}\right] \geq 3$, either $\left|\operatorname{det}\left(L_{2}, u_{2}\right)\right| \geq$ $|D(2,4,5)|=1 / \sqrt{5}>1 / 4$ or $\left|\operatorname{det}\left(L_{2}, u_{2}\right)\right| \geq|D(3,4,3)|=\sqrt{2} / 3>1 / 4$, so we come to a contradiction.

Diagram (4a). Since the diagram $\left\langle u_{6}, u_{1}, u_{3}\right\rangle$ is elliptic, $\left[u_{6}, u_{1}\right] \leq 3$. On the other hand, $L_{2}=\left\langle u_{1}, u_{2}, u_{6}\right\rangle$ is a Lannér diagram, so $\left[u_{6}, u_{2}\right] \geq 7$. This implies that $\left\langle u_{6}, u_{2}, u_{3}\right\rangle$ is a Lannér diagram, which is impossible.

Diagram (4b). Since the diagram $\left\langle u_{6}, u_{2}, u_{3}, u_{4}\right\rangle$ is elliptic, $\left[u_{6}, u_{2}\right] \leq 3$. Hence, $\left[u_{6}, u_{1}\right] \geq$ 7 , and $\left\langle u_{6}, u_{1}, u_{3}\right\rangle$ is a Lannér diagram. This contradiction completes the proof of the lemma.

Lemma 4.15. There are exactly eight compact hyperbolic Coxeter 4-polytopes with 7 facets with Gale diagram $G_{12121}$. Their Coxeter diagrams are shown in the bottom of the second part of Table 4.11.

Proof. Let $P$ be a hyperbolic Coxeter polytope with Gale diagram $G_{12121}$. The Coxeter diagram $\Sigma$ of $P$ contains two Lannér diagrams $L_{1}=\left\langle u_{1}, u_{2}, u_{3}\right\rangle$ and $L_{2}=\left\langle u_{3}, u_{4}, u_{5}\right\rangle$ of order 3 , a dotted edge $u_{6} u_{7}$, and other two Lannér diagrams $L_{3}=\left\langle u_{1}, u_{2}, u_{6}\right\rangle$ and $L_{4}=\left\langle u_{7}, u_{4}, u_{5}\right\rangle$ of order 3. Any subdiagram of $\Sigma$ containing none of these five diagrams is elliptic. Since $L_{3}$ and $L_{4}$ are connected, we may assume that $u_{6}$ attaches to $u_{2}$, and $u_{7}$ attaches to $u_{5}$.

Consider the diagram $\Sigma^{\prime}=\left\langle L_{3}, L_{1}, L_{2}\right\rangle=\Sigma \backslash u_{7}$. Clearly, the only multi-multiple edges that may appear in $\Sigma^{\prime}$ are $u_{1} u_{2}, u_{6} u_{2}, u_{6} u_{1}$, and $u_{4} u_{5}$.

At first, suppose that the edge $u_{6} u_{2}$ is multi-multiple. Then $\left\langle u_{6}, u_{2}\right\rangle$ is not joined with $\left\langle u_{3}, u_{4}, u_{5}\right\rangle=L_{2}$. In particular, $\left[u_{2}, u_{3}\right]=2$, so $\left[u_{1}, u_{3}\right] \neq 2$. Thus, $\left[u_{6}, u_{1}\right]$ is also equal to 2 . Furthermore, since diagrams $\left\langle u_{1}, u_{3}, u_{4}\right\rangle$ and $\left\langle u_{1}, u_{3}, u_{5}\right\rangle$ are elliptic, $\left[u_{3}, u_{4}\right],\left[u_{3}, u_{5}\right]$ and $\left[u_{1}, u_{3}\right] \leq 5$. Therefore, since $\left\langle u_{1}, u_{2}, u_{3}\right\rangle=L_{1}$ is a Lannér diagram, $\left[u_{1}, u_{2}\right] \geq 4$. Now suppose that $\left[u_{1}, u_{4}\right] \neq 2$. Then $\left[u_{3}, u_{4}\right]=2$, so $\left[u_{4}, u_{5}\right] \geq 4$, and the diagram $\left\langle u_{2}, u_{1}, u_{4}, u_{5}\right\rangle$ is not elliptic, which is impossible. The contradiction shows that $\left[u_{1}, u_{4}\right]=2$. Similarly, $\left[u_{1}, u_{5}\right]=2$. Consequently, the diagram $\Sigma^{\prime}$ looks like the diagram shown in Fig. 4.11 , where $m_{45}=\left[u_{4}, u_{5}\right]$. Now we may apply Prop. 3.2: $\operatorname{det}\left(L_{3}, u_{1}\right) \operatorname{det}\left(L_{2}, u_{3}\right)=\cos ^{2}\left(\pi / m_{13}\right)$, where $m_{13}=\left[u_{1}, u_{3}\right]$. Notice that since $\left[u_{1}, u_{2}\right] \geq 4$ and $\left[u_{2}, u_{6}\right] \geq 6$, we have $\left|\operatorname{det}\left(L_{3}, u_{1}\right)\right| \geq$ $|D(2,4,6)|=1$.

If $m_{13}=4$ or 5 , we obtain that $\left[u_{3}, u_{4}\right],\left[u_{3}, u_{5}\right] \leq 3$, which implies $\left[u_{4}, u_{5}\right]=7$ in view of $\left|\operatorname{det}\left(L_{2}, u_{3}\right)\right| \leq \cos ^{2}\left(\pi / m_{13}\right)$. Thus, $\left|\operatorname{det}\left(L_{2}, u_{3}\right)\right| \geq|D(2,3,7)|$. This implies that $\left|\operatorname{det}\left(L_{3}, u_{1}\right)\right| \leq \cos ^{2}(\pi / 5) /|D(2,3,7)|$. An easy calculation shows that in this case 


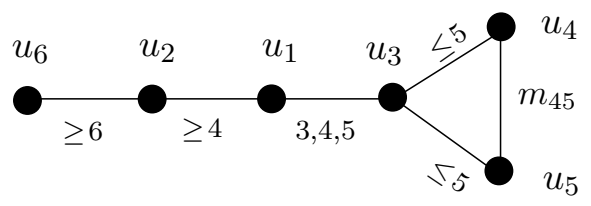

Figure 4.11: A diagram $\Sigma^{\prime}$, see Lemma 4.15

$\left[u_{2}, u_{6}\right] \leq 7,\left[u_{2}, u_{1}\right] \leq 6$. Then we check the finite (small) number of possibilities for $\Sigma^{\prime}$ and see that none of them has determinant equal to zero.

If $m_{13}=3$, then $\left[u_{2}, u_{1}\right] \leq 7$. Therefore, $\left|\operatorname{det}\left(L_{3}, u_{1}\right)\right| \geq|D(2,6,7)|$. Hence, $\left|\operatorname{det}\left(L_{2}, u_{3}\right)\right| \leq \cos ^{2}(\pi / 3) /|D(2,6,7)|$, but such $L_{2}$ does not exist.

The contradiction shows that the edge $u_{6} u_{2}$ is not multi-multiple. Similarly, the edges $u_{6} u_{1}, u_{7} u_{5}$, and $u_{7} u_{4}$ of $\Sigma$ are not multi-multiple either. Thus, the only edges that may be multi-multiple in $\Sigma$ are $u_{4} u_{5}$ and $u_{1} u_{2}$.

Consider again the diagram $\Sigma^{\prime}$ and suppose that the diagram $\left\langle u_{4}, u_{5}\right\rangle$ is not joined with $\left\langle u_{1}, u_{2}, u_{6}\right\rangle$. In particular, this holds if at least one of the edges $u_{4} u_{5}$ and $u_{1} u_{2}$ is multi-multiple. We may apply Prop. 3.1:

$$
\operatorname{det}\left(\left\langle L_{3}, L_{1}\right\rangle, u_{3}\right)+\operatorname{det}\left(L_{2}, u_{3}\right)=1 \text {. }
$$

By definition,

$$
\operatorname{det}\left(\left\langle L_{3}, L_{1}\right\rangle, u_{3}\right)=\operatorname{det}\left\langle L_{3}, L_{1}\right\rangle / \operatorname{det}\left(L_{3}\right) .
$$

We use a very rough bound: $\left|\operatorname{det}\left\langle L_{3}, L_{1}\right\rangle\right|<16$ since it is a determinant of a $4 \times 4$ matrix with entries between -1 and 1 , and $\left|\operatorname{det}\left(L_{3}\right)\right| \geq\left|3 / 4-\cos ^{2}(\pi / 7)\right|=\left|\operatorname{det}\left(\mathcal{L}_{2,3,7}\right)\right|$, since $\operatorname{det}\left(\mathcal{L}_{2,3,7}\right)$ is maximal among all determinants of Lannér diagrams of order 3 . This bound implies

$$
\left|\operatorname{det}\left(L_{2}, u_{3}\right)\right| \leq 1+\left|\operatorname{det}\left(\left\langle L_{3}, L_{1}\right\rangle, u_{3}\right)\right| \leq 1+\frac{16}{\left|3 / 4-\cos ^{2}(\pi / 7)\right|}<261
$$

Now an easy computation shows that $\left[u_{4}, u_{5}\right] \leq 101$. Considering a diagram $\Sigma^{\prime \prime}=$ $\left\langle L_{1}, L_{2}, L_{4}\right\rangle=\Sigma \backslash u_{6}$ in a similar way, we obtain that $\left[u_{1}, u_{2}\right] \leq 101$, too, and we are left with a finite number of possibilities for $\Sigma^{\prime}$ (and for $\Sigma^{\prime \prime}$ ). We list all diagrams $L_{2}$ (less that 1000 possibilities) and all possible diagrams $\left\langle L_{3}, L_{1}\right\rangle$ (less that 10000 possibilities), and find all pairs such that $\operatorname{det}\left(\left\langle L_{3}, L_{1}\right\rangle, u_{3}\right)+\operatorname{det}\left(L_{2}, u_{3}\right)=1$, there are about 50 such pairs. Therefore, we obtain a complete list of possibilities for $\Sigma^{\prime}$ (and for $\Sigma^{\prime \prime}$ ). Then we look for unordered pairs $\left(\Sigma^{\prime}, \Sigma^{\prime \prime}\right)$, such that the diagrams coincide on their intersection, i.e. a subdiagram $\left\langle L_{1}, L_{2}\right\rangle \subset \Sigma^{\prime}$ coincides with a subdiagram $\left\langle L_{1}, L_{2}\right\rangle \subset \Sigma^{\prime \prime}$. There are only 8 such pairs, all them give rise to Coxeter diagrams of Coxeter polytopes. The diagrams are shown in the bottom of the second part of Table 4.11. The weight of the dotted edge is equal to $\sqrt{2} \cos (\pi / 8)$ for the two last diagrams, is equal to $(\sqrt{5}+1) / 2$ for the three diagrams in the second row from the bottom, and is equal to $1+\sqrt{2}$ for the three diagrams in the third row from the bottom. 
Now suppose that the diagram $\left\langle u_{4}, u_{5}\right\rangle$ is joined with $\left\langle u_{1}, u_{2}, u_{6}\right\rangle$. This implies that $\Sigma$ does not contain multi-multiple edges, so we have a finite number of possibilities for the diagrams $\Sigma^{\prime}$ and $\Sigma^{\prime \prime}$. A computation shows that we do not obtain any polytope in this way.

The result of the considerations above is presented below. Recall that there are no 7-dimensional polytopes with 10 facets.

Table 4.8: 8-dimensional polytope with 11 facets

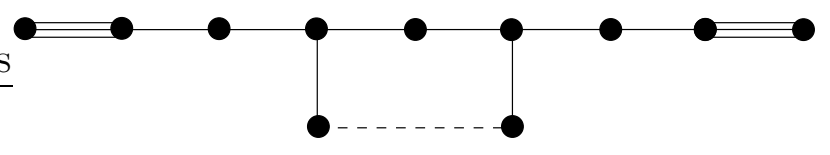

Table 4.9: 6-dimensional polytopes with 9 facets
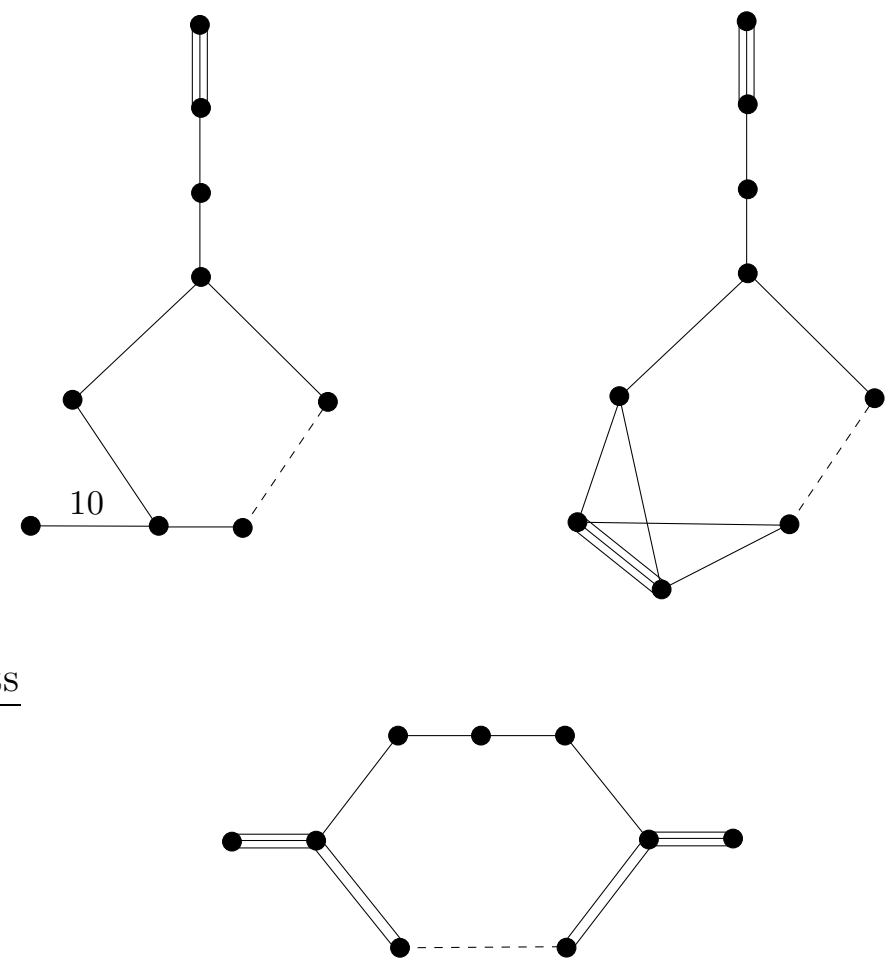
Table 4.10: 5-dimensional polytopes with 8 facets
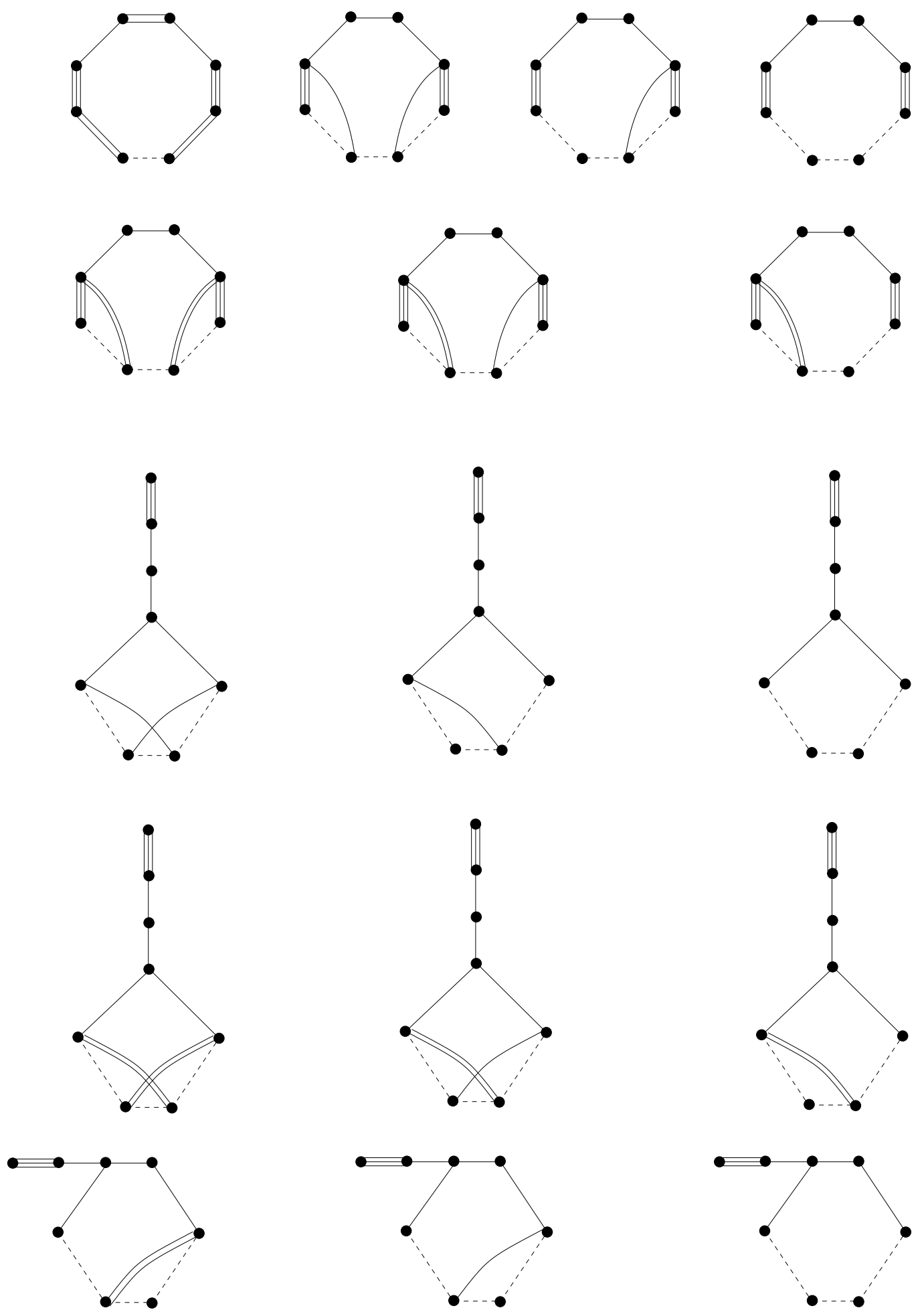
Table 4.11: 4-dimensional polytopes with 7 facets
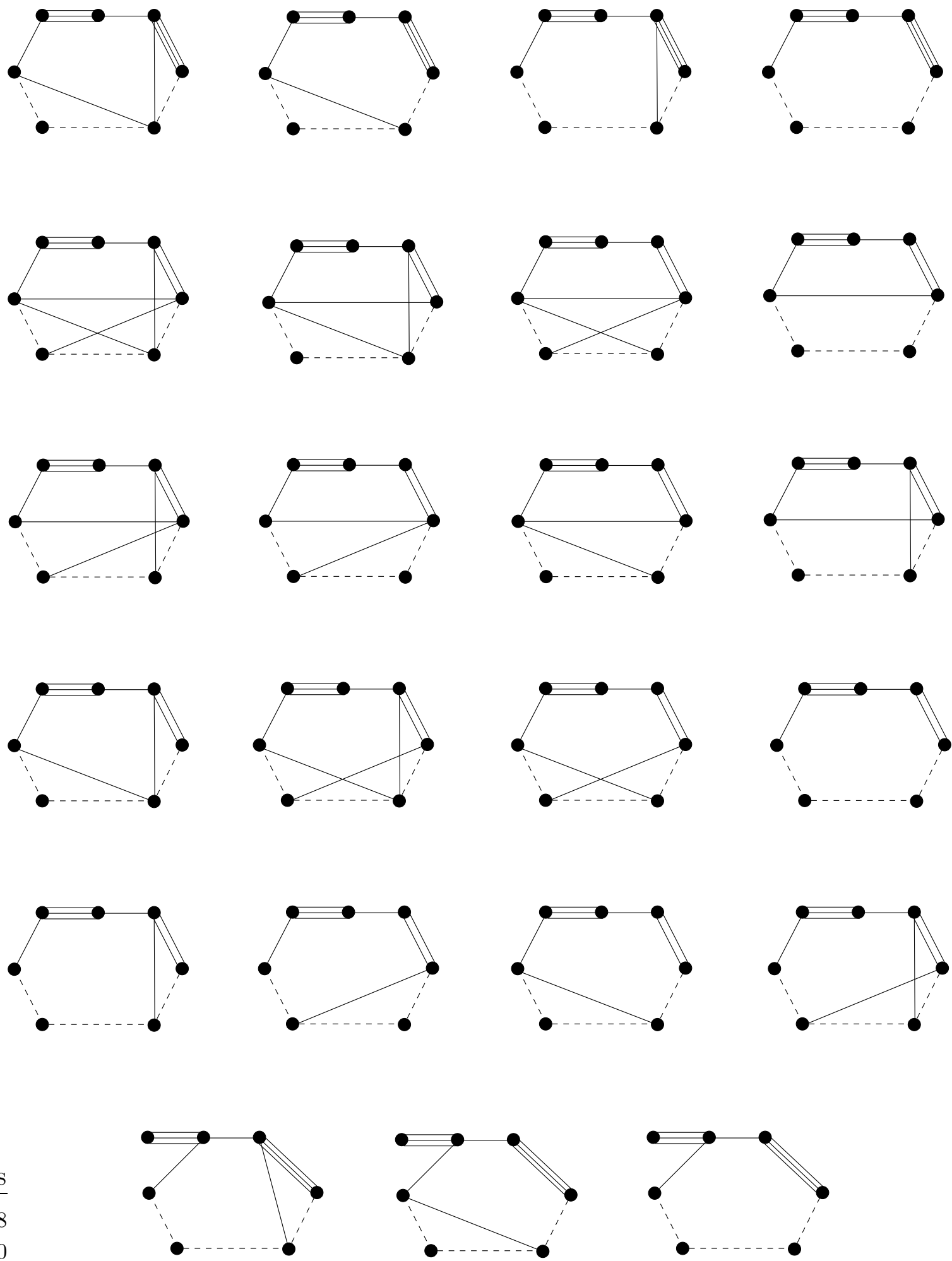
Table 4.11: Cont.
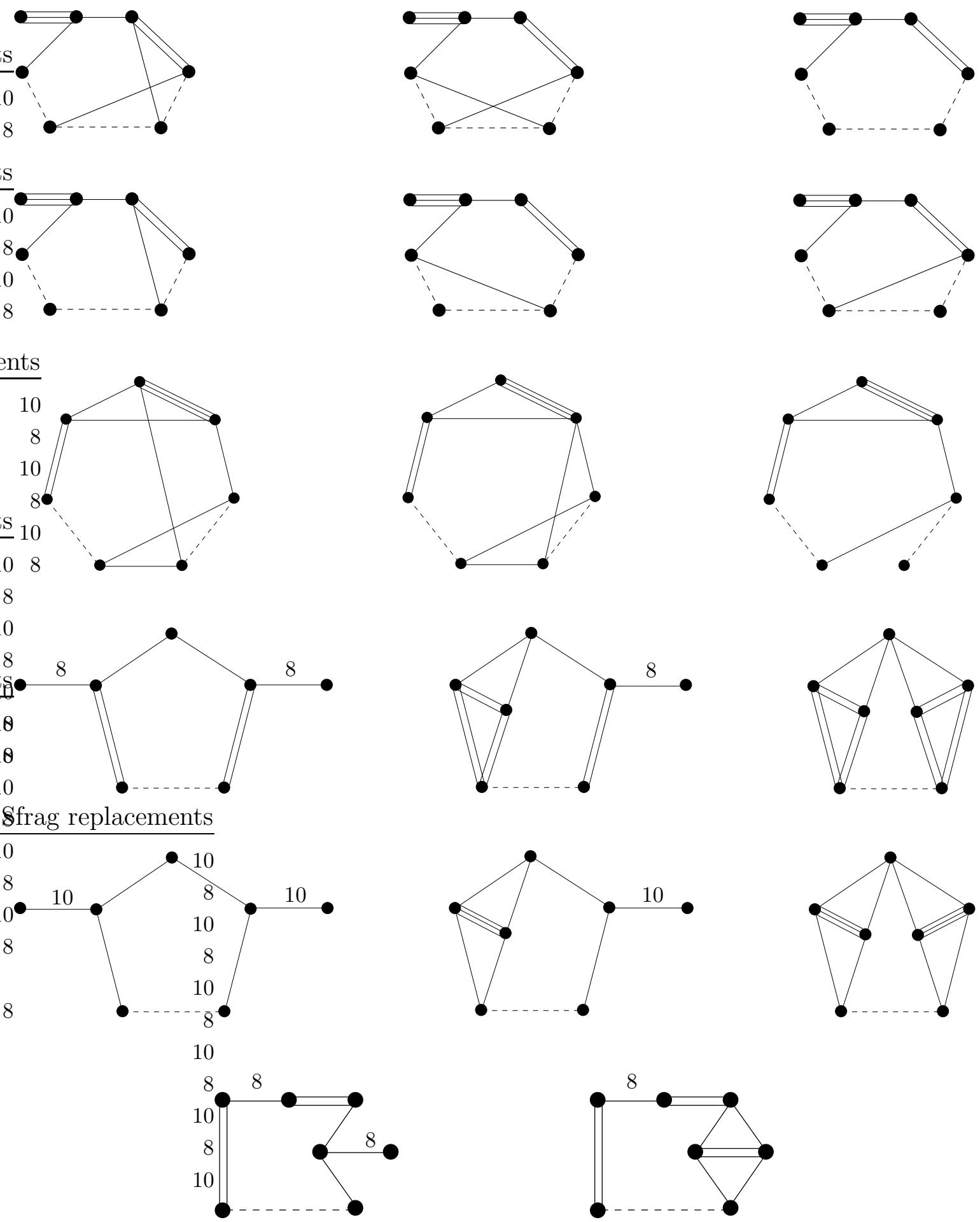


\section{References}

[A] E. M. Andreev, On convex polyhedra in Lobachevskii spaces. Math. USSR Sbornik 10 (1970), 413-440.

[B1] V. O. Bugaenko, Groups of automorphisms of unimodular hyperbolic quadratic forms over the ring $\mathbb{Z}\left[\frac{\sqrt{5}+1}{2}\right]$. Moscow Univ. Math. Bull. 39 (1984), 6-14.

[B2] V. O. Bugaenko, Arithmetic crystallographic groups generated by reflections, and reflective hyperbolic lattices. Advances in Soviet Mathematics 8 (1992), 33-55.

[C] H. S. M. Coxeter, Discrete groups generated by reflections. Ann. Math. 35 (1934), $588-621$.

[E1] F. Esselmann, Über kompakte hyperbolische Coxeter-Polytope mit wenigen Facetten. Universität Bielefeld, SFB 343, Preprint No. 94-087.

[E2] F. Esselmann, The classification of compact hyperbolic Coxeter d-polytopes with d+2 facets. Comment. Math. Helvetici 71 (1996), 229-242.

[FT] A. Felikson, P. Tumarkin, On Coxeter polytopes with mutually intersecting facets. J. Combin. Theory Ser. A (2007), doi:10.1016/j.jcta.2007.04.006.

[G] B. Grünbaum, Convex Polytopes. John Wiley \& Sons, 1967.

[ImH] H.-C. Im Hof, Napier cycles and hyperbolic Coxeter groups. Bull. Soc. Math. de Belg. Série A, XLII (1990), 523-545.

[K] I. M. Kaplinskaja, Discrete groups generated by reflections in the faces of simplicial prisms in Lobachevskian spaces. Math. Notes 15 (1974), 88-91.

[L] F. Lannér, On complexes with transitive groups of automorphisms. Comm. Sem. Math. Univ. Lund 11 (1950), 1-71.

[S] L. Schlettwein, Hyperbolische Simplexe. Diplomarbeit, 1995, Basel.

[P] H. Poincaré, Théorie des groups fuchsiennes. Acta Math. 1 (1882), 1-62.

[V1] E. B. Vinberg, The absence of crystallographic groups of reflections in Lobachevsky spaces of large dimensions. Trans. Moscow Math. Soc. 47 (1985), 75-112.

[V2] E. B. Vinberg, Hyperbolic reflection groups. Russian Math. Surveys 40 (1985), 31-75. 